\begin{tabular}{|l|l|l|l|l|}
\hline Cuadernos I. Geográfica & 25 & pp. 45-84 & Logroño & 1999 \\
\hline
\end{tabular}

\title{
Simuladores de lluvia y su aplicación a la Geomorfología. Estado de la cuestión
}

A. $\operatorname{CERDĀ} \bar{A}^{1}$

RESUMEN: Durante las últimas décadas se ha producido un espectacular desarrollo de los instrumentos utilizados por los geomorfólogos, tanto en el campo como en el laboratorio. De entre estos aparatos destaca los simuladores de lluvia, los cuales se ban utilizado con distintos objetivos desde los años 30. Su utilidad ba revertido en la diversificación en dimensiones, tipos de formadores de gotas, estructuras, caracteristicas de la lluvia, etc. tanto en aquellos simuladores desarrollados a partir de goteadores como los del tipo pulverizador.

En este artículo se repasa la bistoria y la evolución de los simuladores de lluvia y se revisan las ventajas y los inconvenientes de la lluvia simulada, apuntándose los principales usos de la misma: medición de las tasas de erosión del suelo, escorrentia, infiltración, etc. En España la expansión de los simuladores de lluvia se inició con retraso respecto a otros países, aunque en la última década han sido utilizados por la mayoría de grupos de investigación dedicados a la erosión del suelo. Se presenta una amplia bibliografía relacionada con el tema que completa una detallada y exhaustiva revisión de todos los modelos de simuladores de lluvia diseñados, incluyendo entre ellos las modificaciones realizadas por distintos autores sobre los diseños iniciales.

1. Centro de Investigaciones sobre Desertificación-CIDE (CSIC-Universitat de ValènciaGeneralitat Valenciana). Camí de la Marjal, s/n, 46470-Albal, València.

E-mail, acerda@uv.es

Departament de Geografia. Universitat de València. 22060, 46080-València, Spain. 


\section{A. CERDÀ}

ABSTRACT: During the last decades, Geomorphologists have developed a buge variety of scientific instruments as in the field as in the laboratory. Within the invented apparatus stand out the rainfall simulators, which bave been used with different objectives since the 30s. His scientific benefit produced an increase in the variety of sizes, drop-formers, frames, rainfall characteristics, etc., for rainfall simulators based on drop former (tips) and on spray-nozzles systems. This paper reviews the bistory and the evolution of the rainfall simulators, and discusses the advantages and inconvenience of the simulated rainfall. We also point out the main uses: soil erosion rates measurements, runoff, infiltration, etc. In Spain, rainfall simulator expansion started during the 80 s, although during the last decade they have been used for the majority of the soil erosion research groups. A broad literature revision is presented in order to give information of the designed rainfall simulator and the modifications of the original apparatus.

Palabras clave: Simuladores de lluvia, historia, aplicaciones, ventajas, inconvenientes.

Key words: Rainfall simulators, History, Applications, Advantages, Inconveniences.

\section{Introducción}

La razón por la que los investigadores americanos de los años 30 y 40 decidieron utilizar lluvia simulada para realizar mediciones de infiltración, pérdida de suelo y escorrentía está basada en el intento de reproducir el proceso de transformación de la lluvia en caudal, y los procesos anexos: salpicadura, formación de arroyada, desarrollo de costras, etc., y sobre todo infiltración. Hasta ese momento, las mediciones se habían realizado a partir de experimentos que sólo reproducían parcialmente la realidad como los infiltrómetros de cilindro (Hills, 1970) o la inundación de campos de cultivo (Evans et al., 1990). Los experimentos con lluvia simulada difieren con respecto a los anteriores tanto en su mayor complicación técnica como en el concepto general del experimento. La mejora de los simuladores de lluvia con el paso del tiempo ha sido evidente y ello ha permitido que se hayan podido destinar a estudiar diferentes componentes del ciclo hidrológico (infiltración, escorrentía, flujo subsuperficial, salpicadura, lavado de sales, etc.), aunque las relaciones lluvia-escorrentía, infiltrabilidad y pérdida de suelo han sido los aspectos más tratados (Johnston et al., 1980).

El objetivo de este artículo es revisar la evolución de los simuladores de lluvia y aportar información sobre sus características con el fin de facilitar la selección 
SIMULADORES DE LLUVIA Y SU APLICACIÓN A LA GEOMORFOLOGÍA. ESTADO DE LA CUESTIÓN

de los simuladores de lluvia por los grupos de investigadores que pretendan aplicar esta técnica. También se presenta una amplia revisión bibliográfica y un resumen de las características de los simuladores de lluvias diseñados y remodelados por distintos autores.

\section{Origen y evolución de los simuladores de lluvia}

Los simuladores de lluvia nacen en los años 30 y 40 de nuestro siglo, y su origen se encuentra en los problemas metodológicos y conceptuales de las mediciones tomadas con los infiltrómetros de cilindro y con los cálculos de la infiltración a partir de balances hidrológicos. Desde su origen, la simulación de lluvia se ha entendido como una mera herramienta de trabajo, con la cual se pretende reproducir una parte importante del ciclo hidrológico de una forma experimental, acotando la intensidad de la lluvia a los niveles deseados.

La espectacular evolución e innovación de esta técnica durante la segunda mitad del siglo XX, se inició como consecuencia de la importante investigación agronómica llevada a cabo en USA durante los años de entreguerras. En este país, se inició la construcción de simuladores en los años 30, se continuó en los 40 , y se aceleró en los 50 (Neff, 1979), aumentando continuamente el número de simuladores y apareciendo nuevos diseños a partir de los años 60. Los pioneros en esta técnica fueron los estudiosos de la erosión del suelo (Nichols y Sexton, 1932; Neal, 1938), pero rápidamente sus datos y sus protocolos experimentales fueron recogidos por estudiosos del proceso de infiltración (Marsh, 1939; Beutner et al., 1940). A este primer uso a escala de parcela le sucedió una aproximación basada en las cuencas experimentales de laboratorio para el estudio de la hidráulica de la escorrentía (Christiansen, 1941; Izzard, 1944; Holtan y Kirkpatrick, 1950; Turner, 1965; Chow y Yen, 1974), y de la infiltración (Holtan, 1961).

En la actualidad, aquellos primeros simuladores de lluvia parecen instrumentos rudimentarios. Pero, las deficiencias de aquellos aparatos, en contra de lo que podíamos pensar, no se debieron a la falta de recursos técnicos para su construcción, sino a la falta de conocimientos sobre las características físicas de la lluvia. Las herramientas y materiales necesarios para construir un simulador de lluvia como los actuales, ya existían en 1930 con la excepción de los ordenadores que permiten la automatización de los registros de las medidas con gran facilidad.

Los simuladores de lluvia construidos antes de la segunda guerra mundial trataban sólo de aplicar una cantidad de agua determinada sobre una superficie, sin tener en cuenta la forma en que el agua se ponía en contacto con el suelo. Las publicación de las características de la lluvia (Laws, 1941; Spilhaus, 1948), y estudios específicos sobre la relación entre la intensidad y el tamaño de las gotas (Laws y Parsons, 1943; Gunn y Kinzer, 1949), hicieron que los investigadores mejorasen los simuladores, teniendo siempre a la lluvia natural como modelo a imitar. 
Los estudios de los años 40 fueron sólo el inicio, ya que será en las siguientes dos décadas cuando el conocimiento de las características de las lluvias naturales avanzó notablemente (Jones, 1959; Hudson, 1963), y el reconocimiento de su importancia sobre la pérdida de suelo (Smith y Wischmeier, 1962) producirá una aceleración en el desarrollo de los simuladores de lluvia (Figuras 1 y 2). A esto, también ayudará el desarrollo de ecuaciones e índices de erosión extraídos a partir de estudios experimentales (Wischmeier, 1959; Chow y Harbaugh, 1965; Wischmeier y Smith, 1978; Wilson y Rice, 1987), en los que los propios simuladores de lluvia fueron de gran utilidad.

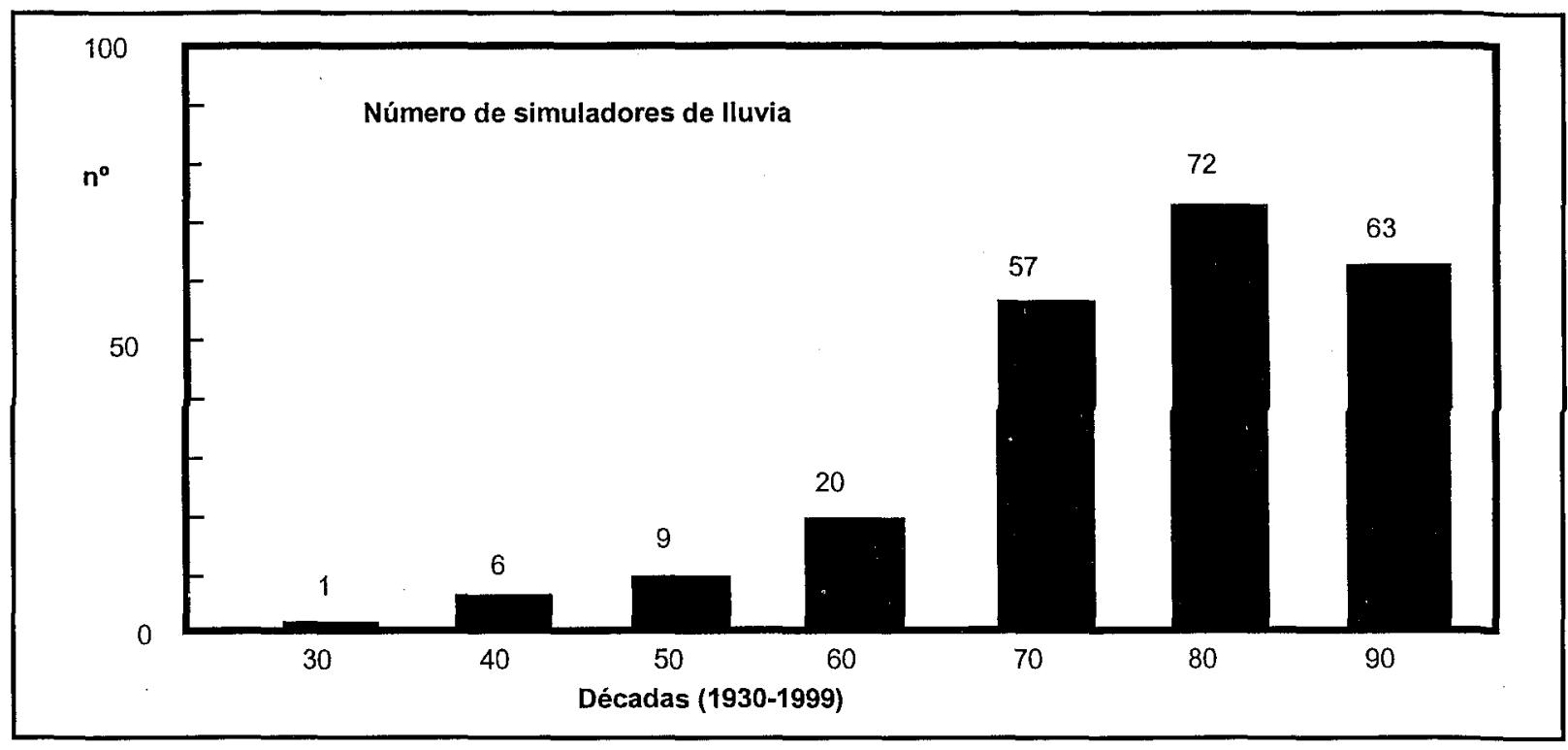

Figura 1. Número de simuladores de lluvia diseñados por décadas.

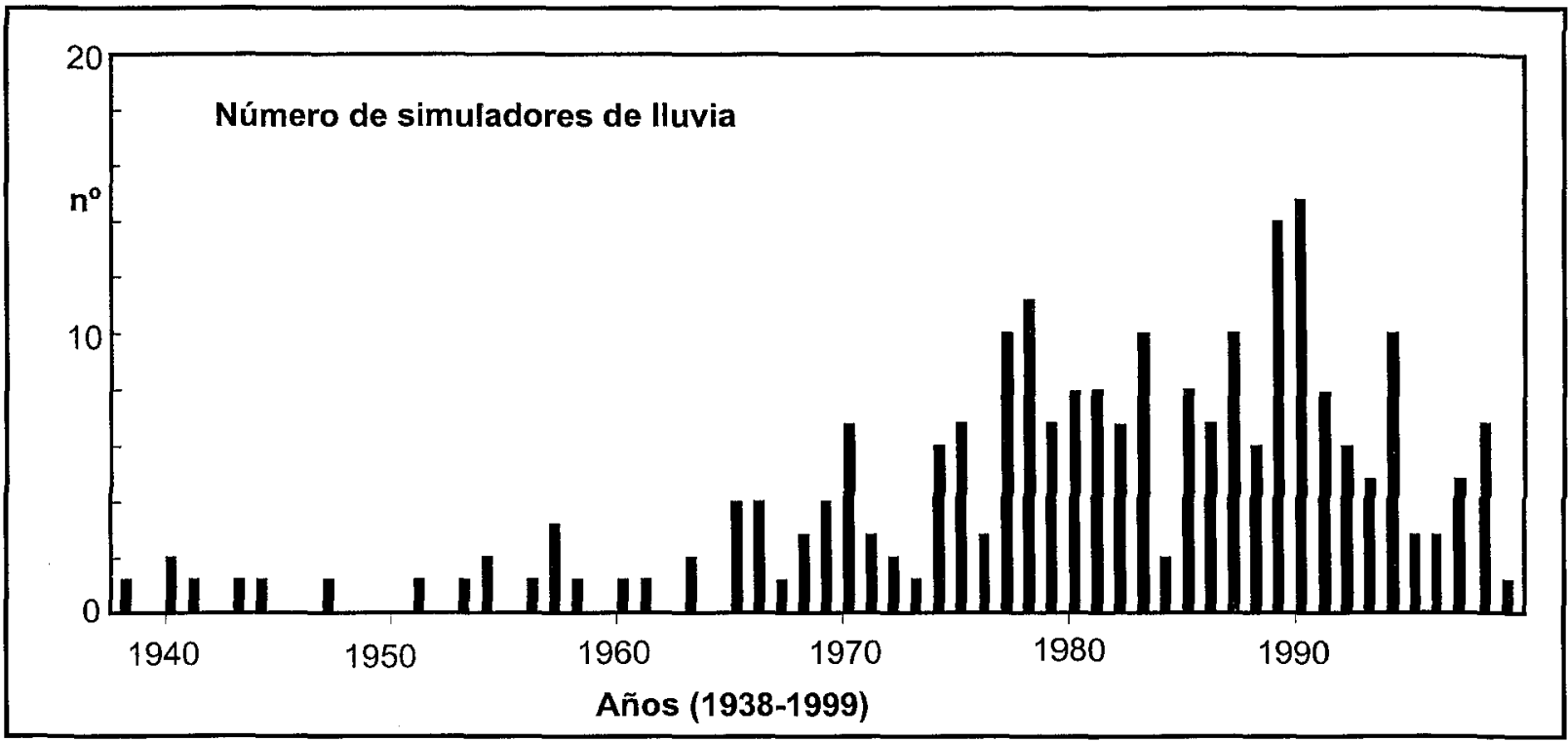

Figura 2. Número de simuladores de lluvia diseñados por años. 
Los estudios anteriormente mencionados dan una gran importancia a la energía cinética de las gotas de lluvia en los procesos de erosión y en el sellamiento de la superficie del suelo. Por ello, a partir de los años 60 uno de los grandes retos de los investigadores será construir simuladores de lluvia que imiten, no sólo la distribución del tamaño de las gotas de lluvia, sino que además éstas precipiten con una energía cinética semejante a la real. Los primeros simuladores desarrollados en USA fueron copiados, remodelados e innovados en otros países a partir de los años 60. Rose (1960) en Uganda, Kazó (1966) en Hungría y Steinhart (1966) en Israel desarrollaron los simuladores de tipo goteador a partir de los ya desarrollados anteriormente en laboratorios norteamericanos. Allí, en concreto en el USDA (United State Department of Agriculture) ya se estaban utilizando grandes simuladores de lluvia del tipo pulverizador (Meyer y McCune, 1958), pero estos autores aún copiaron los simuladores goteadores iniciales.

Las siguientes dos décadas, años 70 y 80 , se caracterizan por la proliferación de simuladores, no sólo en EE:UU, como había ocurrido en las décadas anteriores, sino también en el resto del mundo. En ese momento es cuando llega a España esta técnica (Sanroque et al., 1984; Benito et al., 1986; Calvo et al., 1988; Navas, 1988; Pelegrin, 1989). La expansión geográfica de esta herramienta de trabajo es fruto, sin duda, de la ampliación del número de disciplinas que utilizan los simuladores de lluvia. Es de destacar la pérdida del monopolio que habían mantenido los ingenieros agrónomos durante las primeras décadas, así como el auge que ha tenido esta técnica entre hidrólogos y geomorfólogos, siendo además estos últimos los que se han mostrado más innovadores en sus diseños.

Otra característica de la evolución sufrida por los simuladores de lluvia en los últimos veinte años ha sido la especialización de cada tipo de simulador en unos temas determinados de la investigación. Hasta los años 60, la única especialización de los instrumentos, y no siempre, se reducía a ser dedicados a estudios de laboratorio o bien a investigación de campo. Esto se debe a que eran utilizados sólo en campos de cultivo, y por ello todos repetían el mismo esquema: grandes aparatos, difíciles de manejar y excesivamente costosos (Meyer, 1960). En cambio, en las últimas décadas, con la entrada de nuevas disciplinas como la Ecología, Hidrología, Edafología, y especialmente la Geomorfología, se produjo una diversificación de los diseños, ya que también son diversos los objetivos (Lane, 1986).

La creación de nuevos simuladores de lluvia, o bien la modificación de antiguos diseños, es muy frecuente. Las razones antes argumentadas -adaptación de los diseños a los objetivos de los estudios- influyen en esto, pero además, hay que tener en cuenta que cada vez hay más estudios sobre la lluvia natural de regiones concretas (Carter et al., 1974), lo cual produce continuas modificaciones de los simuladores con el fin de imitar mejor la lluvia natural de espacios geográficos específicos (Bubenzer et al, 1979). 


\section{Tipos de simuladores de lluvia}

Hay dos grandes grupos de simuladores de lluvia. En uno las gotas de lluvia se precipitan de forma natural o a muy baja presión (goteadores), mientras que en los otros las gotas se generan a partir del paso de agua a presión por las boquillas (pulverizadores). Estas diferencias suponen características distintas de las gotas de lluvia y además determinan las características del aparato que en el primero de los casos hace que sea muy difícil su uso en el campo (de Ploey, 1983).

\subsection{Simuladores goteadores}

Los primeros simuladores de lluvia que se construyeron fueron de tipo goteador. Aunque su construcción y uso se produjo durante los años 30, la publicación de los primeros diseños y de su resultado no llegó hasta pasada una década. Estos aparatos se caracterizan por su reducida complicación técnica, ya que consisten en formar gotas individuales y dejarlas precipitar desde ciertas alturas. Los primeros modelos utilizaban fibras de algodón para conseguir el goteo, siendo el primer simulador de lluvia de este tipo diseñado por Ellison en los años 30 (Ellison, 1947).

Estos modelos, a pesar de su escasa precisión, sufrieron una continua sofisticación que les llevó a complicaciones técnicas tales como el incremento del tamaño de las gotas con la colocación de fibras de algodón más gruesas (Rowe, 1940). De todos los construidos, sólo uno de estos modelos (Barnes y Costel, 1957) está todavía en funcionamiento actualmente (Peterson y Bubenzer, 1984).

El origen y desarrollo de los simuladores de lluvia goteadores fue paralelo al de dos temas de gran interés en la época. Por una parte los estudios sobre las características físicas de la lluvia (Laws, 1941) que confirmó la reducida precisión de la formación de gotas mediante hilos, lo que llevó a buscar otra técnica para producir las gotas artificiales: agujas hipodérmicas, finos tubos de vidrio, polietileno o acero, etc., para producir gotas artificiales de mayor tamaño y desde mayores alturas. Por otra, el estudio de la estabilidad de los agregados del suelo mediante el golpeteo de una o varias gotas sobre los agregados. Para ello se desarrollaron buretas que producian gotas de un calibre conocido, con posibilidades de cambiar el tamaño de las gotas y la frecuencia del golpeteo (McCalla, 1994; Low, 1954; Rai et al., 1954; Pereira, 1956). Una vez desarrollados estos formadores de gotas -uno o un conjunto de ellos- se pueden entender como un simulador de lluvia, aunque muy primitivo (Figura 3).

Debido a las características de este tipo de simulador: fragilidad de algunos de sus componentes, problemas de transporte y mantenimiento, difícil instalación, etc., ha sido utilizado generalmente en investigaciones abordadas en laboratorio (Mutchler y Moldenhauer, 1963), aunque ha habido casos en los que sus componentes se han adaptado para su uso en el campo. 


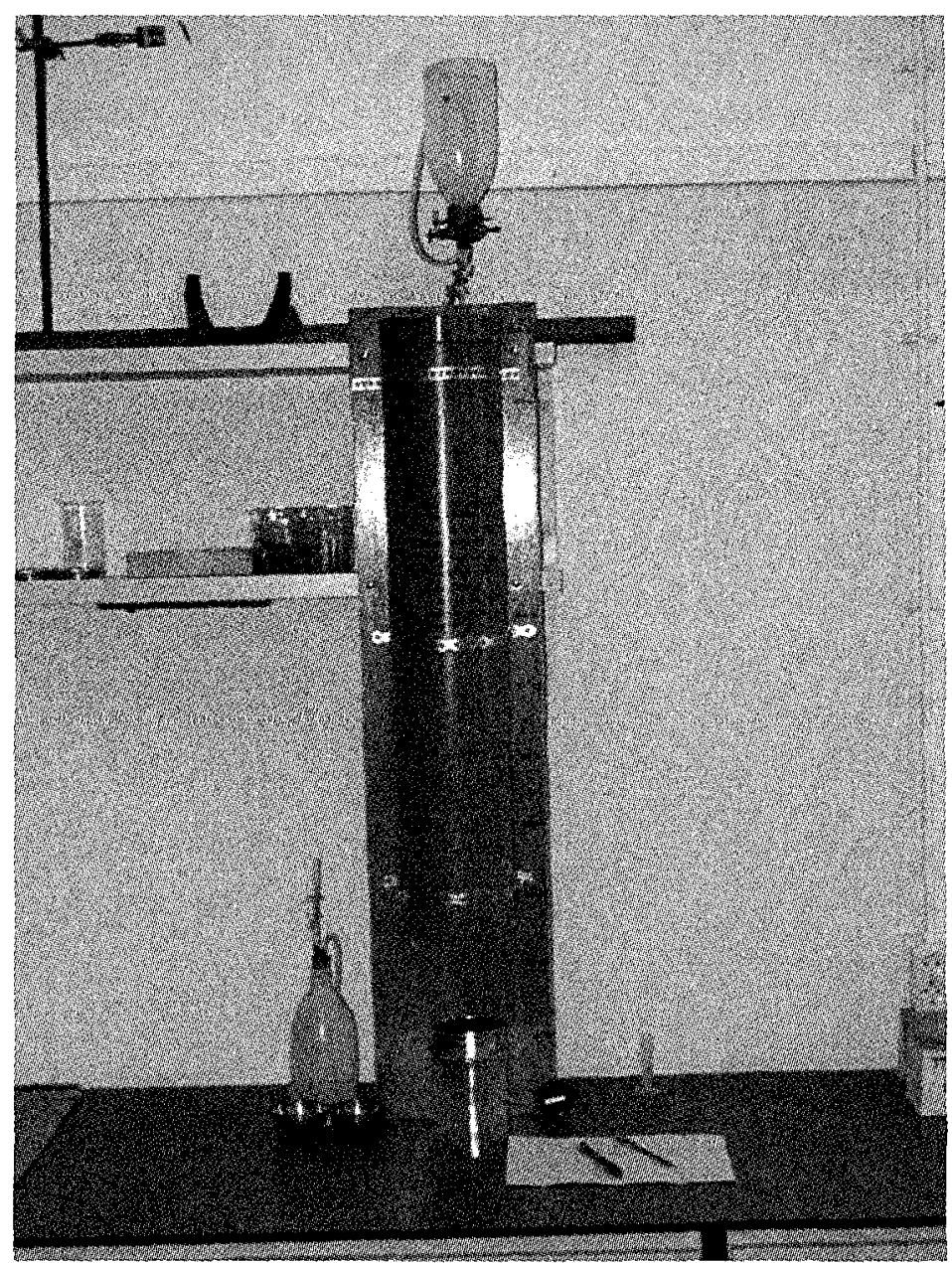

Figura 3. Formador de gotas mediante bureta utilizado para la cuantificación de la estabilidad de agregados (Imeson y Jungerius, 1976; Cerdà, 1998).

En general, los simuladores goteadores tienen un fácil control sobre las características de la lluvia por medio de técnicas como la separación de las agujas, la variación en el tamaño de éstas (Mutchler y Hersmeier, 1965), o bien el cambio en la presión del agua del depósito superior (Munn y Hungtinton, 1973). Para mejorar la producción y control de lluvia artificial se idearon sistema de interrupción del paso del agua en la parte superior de cada aguja (Römkems et al., 1975)

Los tamaños de las gotas en estos instrumentos son muy dispares, ya que cada investigador intenta imitar una lluvia diferente: $2,2 \mathrm{~mm}$ de tamaño medio en Bubenzer y Jones (1971), o bien 5,6 mm en Adams et al., (1957). La desventaja principal de los simuladores goteadores es que en ellos la lluvia simulada no alcanza la energía cinética de la lluvia natural, ya que para esto, los formadores de gotas deberian estar colocados a unos $20 \mathrm{~m}$ de altura para los tamaños de gota mayores (Epema y Riezebos, 1983). Esto explica su reducida utilización en trabajos de campo, donde alturas mayores a $3 \mathrm{~m}$ son muy difíciles de manejar. 
Otro de los problemas de los simuladores goteadores es que el impacto de las gotas de lluvia se produce siempre en el mismo punto, perdiéndose así una de las características de la lluvia natural, la estocasticidad. Este problema se ha solucionado en algunos simuladores con el movimiento continuo de éstos (Kleijn et al., 1979; Poesen, 1984), o bien con la colocación de mallas móviles por debajo de las agujas (Imeson et al., 1982), lo cual produce el mismo efecto que el movimiento del simulador, pero con menor complicación técnica. Esta última innovación es, en cierto aspecto, una vuelta a las tiras de algodón de los primeros simuladores goteadores.

Una última deficiencia de este tipo de simuladores es que las gotas producidas, en casi todos ellos, son del mismo tamaño. Hay investigadores (Brakensiek et al., 1979) que han logrado producir gotas de diferentes calibres en un simulador goteador con la utilización de compresores de aire alrededor de los formadores de gotas, o bien mallas sobre las que se rompen las gotas con los cuales también se conseguía la estocasticidad.

Generalmente, estos simuladores son de difícil manejo por el depósito de agua elevado que deben de tener, por la necesidad de montarlos a gran altura y por el propio consumo de agua. Estos inconvenientes, unidos a las dificultades en el transporte, han agudizado el ingenio de los investigadores, llegándose a construir simuladores que son transportables y utilizables incluso por un operador (McQueen, 1963; Blackburn et al., 1974), aunque en general son de manejo más complicado que los simuladores pulverizadores.

En las dos últimas décadas, se ha producido un aumento en la construcción de simuladores goteadores muy sencillos con dedicación exclusiva para trabajos específicos de laboratorio. La razón de esto es que en algunos tipos de experimentos no hace falta conseguir imitar la lluvia natural, sólo conocer la entrada en el sistema suelo de una determinada cantidad de agua (Hino et al., 1987; 1988). Una característica muy particular de los simuladores de laboratorio es que en ocasiones sólo son parte de otro simulador mayor, aprovechándose de las grandes posibilidades que ofrece la precisión del trabajo en laboratorio: electricidad, inexistencia de viento, paredes que pueden funcionar como soporte, etc. (Römkens, 1979).

Estas ventajas de los laboratorios hacen que los simuiadores puedan ser mucho más sencillos que los utilizados en el campo, ya que un simple montaje de una bureta en alto con diferentes capilares para poder crear gotas de diferentes tamaños puede ser considerado como un simulador de lluvia (Rai et el., 1954; Pereira, 1956; Moldenhauer, 1965; Epema y Riezebos, 1983; Imeson, 1976).

El creciente interés del mundo científico por el conocimiento de la respuesta de los suelos in situ, ha favorecido la construcción de simuladores portátiles, entre los que destaca el modelo de Adams et al., (1957), el cual tiene 100 formadores de gotas de $5,5 \mathrm{~mm}$ y está colocado a $1 \mathrm{~m}$ de altura, siendo la energía cinética de estas gotas la misma que la de la velocidad terminal de una gota de $3,44 \mathrm{~mm}$. 
Gran parte de los simuladores de lluvia tienen su origen en estudios específicos de laboratorio. Un ejemplo de esto es el S.T.O.R.M.-1, simulador ideado y dedicado a estudios de laboratorio sobre la importancia del splash en la erosión, y la influencia sobre ésta del ángulo de la ladera (De Ploey; 1981; Moeyersons, 1983). Este aparato está situado a 7,1 m de altura y las gotas formadas son de 4,1$4,2 \mathrm{~mm}$, alcanzando el $90 \%$ de la velocidad terminal, pero tras ser dispersadas por una malla colocada a $5,4 \mathrm{~m}$ de altura el tamaño medio se reduce a $2,5 \mathrm{~mm}$.

Hay simuladores utilizados en el campo y en el laboratorio, situación ideal por otra parte para poder comparar resultados. Este es el caso del simulador diseñado por Imeson (1977), el cual ha sido utilizado en distintos ambientes: templados (Riezebos y Seyhan, 1977), semiáridos (Imeson et al., 1992) y tropicales (Wierda et al., 1989) (Figura 4). Aunque no es lo mas usual en el caso de los goteadores, esta situación de simuladores de utilización doble, laboratorio-campo, ya se producía en los años 60 (Steinhardt y Hillel, 1966). También se han desarrollado simuladores de muy reducido tamaño dedicados exclusivamente a estudios en el laboratorios (Kamphorst, 1987).

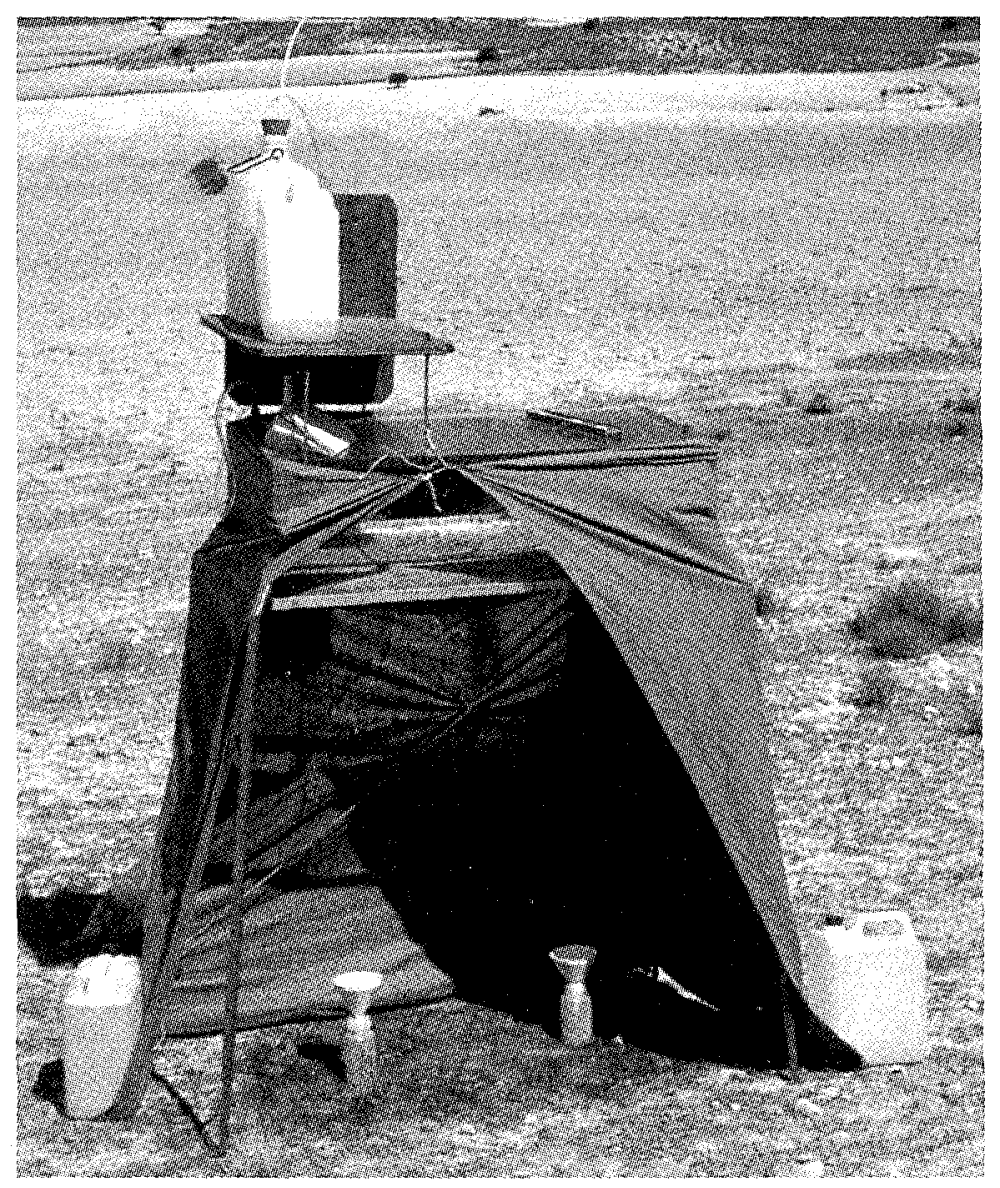

Figura 4. Simulador de lluvia tipo goteador en el campo (Imeson, 1977). 
A. CERDÀ

\subsection{Simuladores pulverizadores}

Durante la década de los 40, y especialmente los 50 y 60 , aparecieron los simuladores de lluvia del tipo pulverizadores, los cuales vieron incentivado el crecimiento de su número cuando se comprobó que una de las características de la lluvia natural es su elevada energía cinética.

El sistema productor de la lluvia en estos instrumentos es a partir del paso del agua a presión por una boquilla que dispersa o pulveriza el agua. Excepto en los casos de simuladores construidos con un gran capital (Meyer y McCune, 1958), las boquillas provienen de diseños dedicados a la irrigación, extínción de incendios, o bien dedicadas a simular la lluvia, pero siempre con fines agronómicos.

La primera generación de simuladores pulverizadores se inició con la aparición de la boquilla tipo $F$ (Wilm, 1943). El desconocimiento de las características físicas de la lluvia, llevó a que las boquillas se orientasen hacia arriba, perdiendo de esta forma toda la energía cinética complementaria fruto de la velocidad inicial de las gotas. En general, el uso de este diseño se encaminó a estudios de infiltrabilidad de los suelos, en los que la energía cinética tiene una importancia secundaria cuando los suelos están bien vegetados (Horton, 1939; Beutner et al.; 1940; Holtan y Kirkpatrick, 1950; Leathan y Riesbol, 1950). A pesar de esto, las boquillas tipo $F A$ y $F$, son utilizadas durante algunos años con sensibles modificaciones de los accesorios hasta la década de los 70 (Dortignac, 1951; Parcker, 1957; Meewing, 1969).

Una segunda generación de simuladores fue fruto de la efervescencia de estudios sobre conservación de suelo en los años finales de la década de 1950 en EE.UU., lo que hizo posible la aparición del primer simulador de lluvia con la suficiente energía cinética para compararse a la precipitación natural. El rainulator, nombre que le dan sus mismos autores (Meyer y McCune, 1958), tenía las boquillas orientadas hacia abajo, caracterizándose el soporte por su aparatosidad y elevado coste, lo cual se compensa con su enorme utilidad sobre parcelas grandes $\left(75 \mathrm{~m}^{2}\right)$ y su capacidad para reproducir lluvias con energía cinética cercana a la natural (Meyer, 1960). Toda la mejora, además de en el tamaño, residió en el diseño de la boquilla ( 80100 Veejet), la cual fue elegida porque el tamaño de las gotas y la erosividad de las tormentas creadas con ella estaban muy cerca de las lluvias naturales del medio oeste americano. En el primer modelo de rainulator se consiguieron tamaños de gotas grandes, pero como consecuencia de esto, las intensidades de lluvia eran excesivas.

La colaboración de uno de las constructores del rainulator con otros investigadores aportó nuevas soluciones. Una de éstas fue la del movimiento alternativo de las boquillas (Bubenzer y Meyer, 1965), la cual se realizó primero en un simulador de laboratorio y ha sido mantenida incluso en simuladores de reciente construcción (Asseline y Valentin, 1978; Foster et al., 1979). Meyer et al., (1979) 
desarrollaron un sistema de movimiento de las boquillas más rápido sobre parcelas de reducida extensión, lo que junto a otras mejoras ha posibilitado que los ciclos de intermitencia de 20 segundos se hayan reducido a tan sólo medio segundo (Foster et al., 1982; Zegelin y White, 1982).

Las modificaciones del diseño original del rainulator han sido múltiples, ya que algunos autores han utilizado parcelas de $8,4 \mathrm{~m}^{2}$ y han cambiado las unidades de propulsión del simulador para reducir la intermitencia (Siemens y Oschwald, 1978). Las modificaciones del rainulator han afectado incluso a las boquillas, siendo sustituida la 80100 por la 80150 veejet, ya que en esta última, la energía cinética es mucho mayor (Meyer y Harmon, 1979) (Figura 5). Pero esto, no ha reducido la intermitencia, ni la imposibilidad de su uso en zonas en pendiente, ni su complejo diseño (Foster et al., 1979).

La tercera generación de simuladores pulverizadores surgió con la intención de reducir la aparatosidad y costes del rainulator. La primera solución para esto fue la de Swanson (1965), la cual consistía en acoplar las boquillas a un aspersor de molinete móvil para producir lluvia simulada sobre las mismas parcelas del rai-

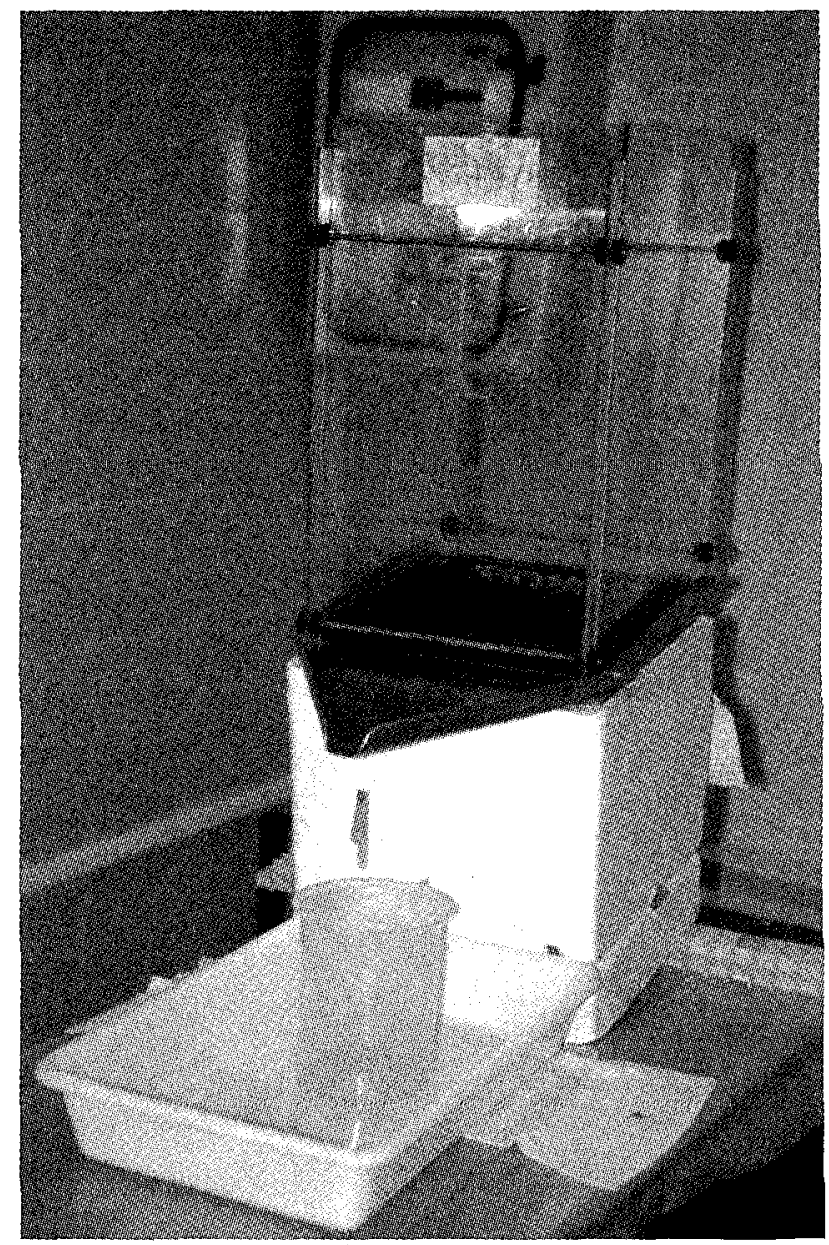

Figura 5. Simulador de laboratorio del tipo goteador (Kamphorst, 1987). 
nulator. La mejora en la transportabilidad del simulador de Swanson fue una obsesión entre los colaboradores del autor y en diseños posteriores (Auerswald, 1986; Simanton et al., 1986) (Fig. 6).

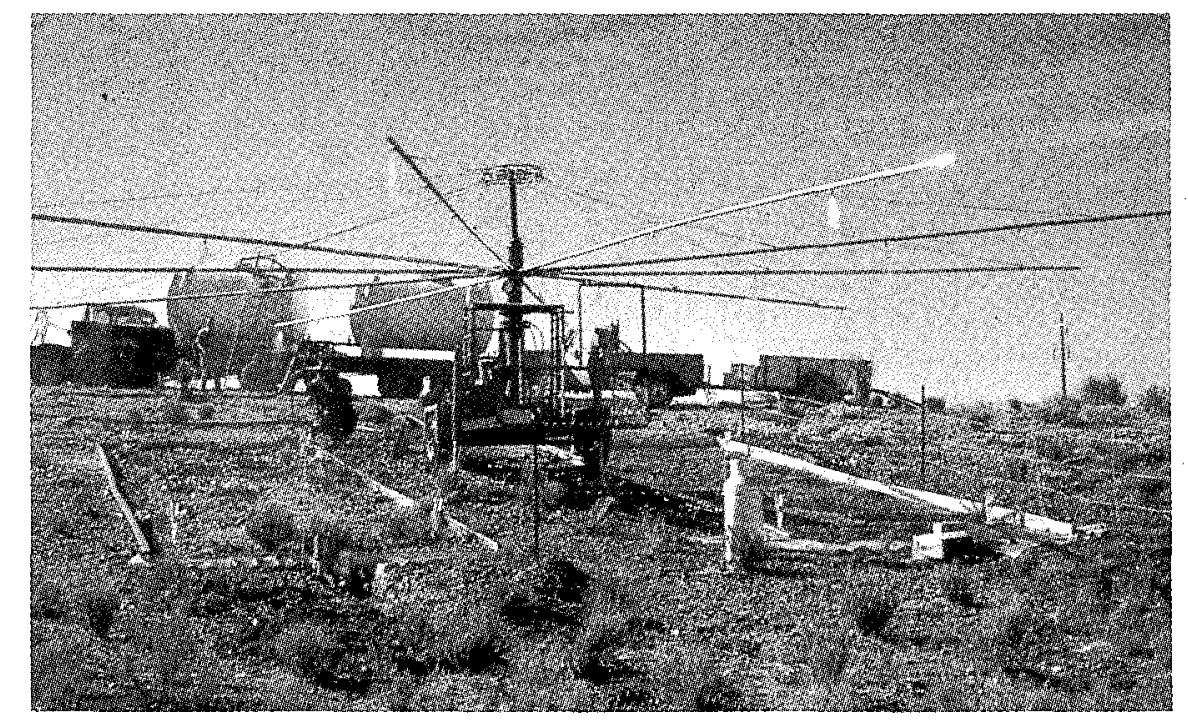

Figura 6. El "Rotatin boom rainfall simulator "desarrollado por Swanson (1965) y modificado por distintos autores. Fotografía de J.R. Simanton.

A pesar de la mejora en la manejabilidad de los simuladores de la tercera generación, todos ellos estaban diseñados para estudios agronómicos, donde se podían utilizar grandes instrumentos sobre amplias y llanas superficies. Con la cuarta generación de simuladores de lluvia por pulverización, se pasa a trabajar con instrumentos más pequeños y manejables, dedicados al estudio de ambientes poco accesibles. Entre ellos, el Infiltrómetro dispersador de Purdue fue el diseño más conocido (Bertrand y Parr, 1961), aunque sólo alcanza el 60 \% de la energía cinética de la lluvia natural. Las continuas modificaciones del diseño original han hecho que este simulador sea aún utilizado en la actualidad, estando dedicado sobre todo al estudio del proceso de infiltración (Shriner et al., 1977; Martínez et al., 1979).

La reducción de la intensidad de la lluvia simulada -tan interesante para los estudios de infiltración- fue una de las obsesiones de los investigadores que utilizaban instrumentos de pequeño tamaño durante la década de los 60 . Sobre esta idea nace la quinta generación de simuladores, la cual se caracteriza por el uso de discos cortadores de lluvia, que facilitan la producción de gotas de tamaños grandes con bajas intensidades de lluvia (Morin et al., 1967). En este caso, la boquilla seleccionada, produce gotas de $2,6 \mathrm{~mm}$ de tamaño medio $\left(\mathrm{D}_{50}\right)$, funciona continuamente, y afecta a una superficie de $2 \mathrm{~m}^{2}$, aunque la parcela sólo ocupa $1 \mathrm{~m}^{2}$. El suelo sólo recibe lluvia de forma intermitente debido a que por debajo 
de la boquilla se coloca un disco rotatorio con una muesca que corta el cono de lluvia y controla la intensidad de la lluvia (Figura 7 y 8).

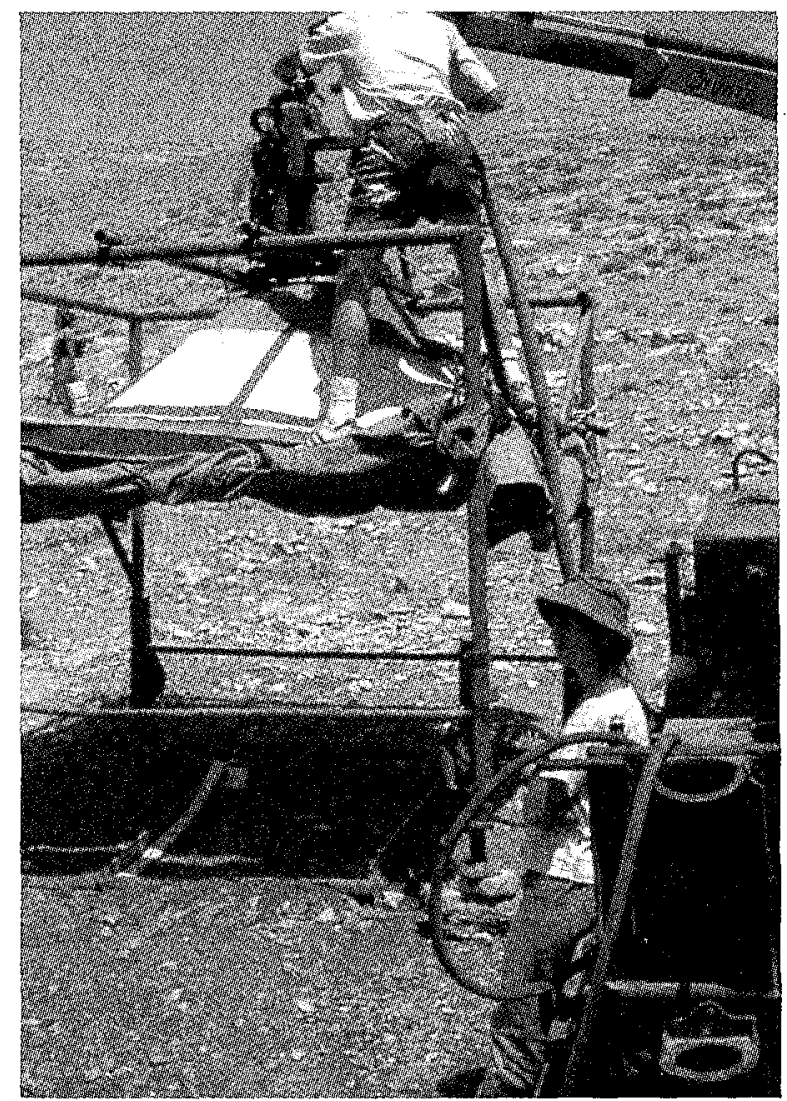

Figura 7. Vista de un simulador de lluvia pulverizador con disco controlador de la intensidad de lluvia (Morin et al., 1967). Fotografía H. Lavee.

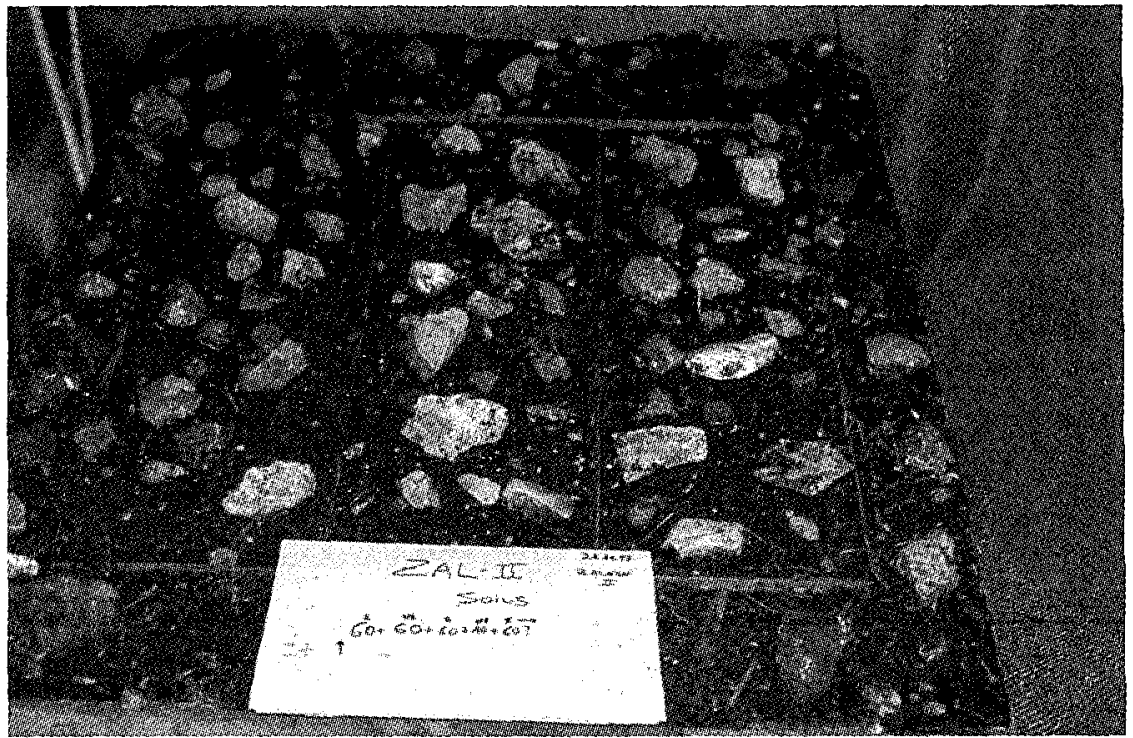

Figura 8. Parcela utilizada en el laboratorio por el simulador de lluvia Morin et al., (1967). 
La mayor transformación del disco rotatorio de Morin se introduce con la modificación de la ranura original, la cual es sustituida por cuatro de menor calibre (Amerman et al., 1970). Esto producirá que el impacto de la lluvia se produzca cada 0,25 segundos, lo cual es imperceptible para el ojo humano. Con esta innovación Amerman modificó el simulador infiltrómetro de Pardue (Bertrand y Parr, 1961), el cual anteriormente ya había sufrido diferentes cambios y renovaciones (Dixon y Petersen, 1968).

Las mejoras en los discos y en la transportabilidad de los aparatos han sido muy importantes (Ratwitz et al., 1972; Gierson y Oades, 1977; Marston, 1982), aunque la modificación más conocida es la llevada a cabo por Bubenzer et al., (1979), en la que se usa una boquilla del tipo 1/4 MH 14 WSQ con el fin de reducir el tamaño de las gotas e imitar la lluvia de la región de Paulose (USA). La expansión geográfica de este tipo de simuladores ha sido muy importante llegando incluso a los países sudamericanos (Rodríguez, 1997).

Tras el desarrollo de cinco fases en la construcción de simuladores de lluvia se inició una diversificación en los diseños que se inicia a finales de los años 60 . Esto supuso la aparición de la sexta generación, la cual no tiene una característica común, excepto que el diseño está de acuerdo con los objetivos de la investigación y se tiende a una mejora en la flexibilidad de los componentes del instrumento y su versatilidad (Steinhart y Hillel, 1966; Anderson et el., 1968; Holland, 1969; Ponce, 1975; Ponce and Hawkins, 1978; Johnston et al., 1980; Bork, 1981; Hirschi et al., 1981; Hodges y Bryan, 1982; 1982; Parcker y Schumm, 1982; Scoging, 1982; Luk et al., 1986; 1987; Tossell et al., 1987; Calvo et al; 1988; Becher, 1990). Diseños de esta sexta generación son muy abundantes, ya que muchos autores han modificado parcialmente antiguos aparatos (cambios de estructura, protectores de viento, etc.) para adecuarlos a nuevos temas de investigación (Cerdà ét al., 1997) (Figuras 9 y 10). En otros casos, los diseños son com-
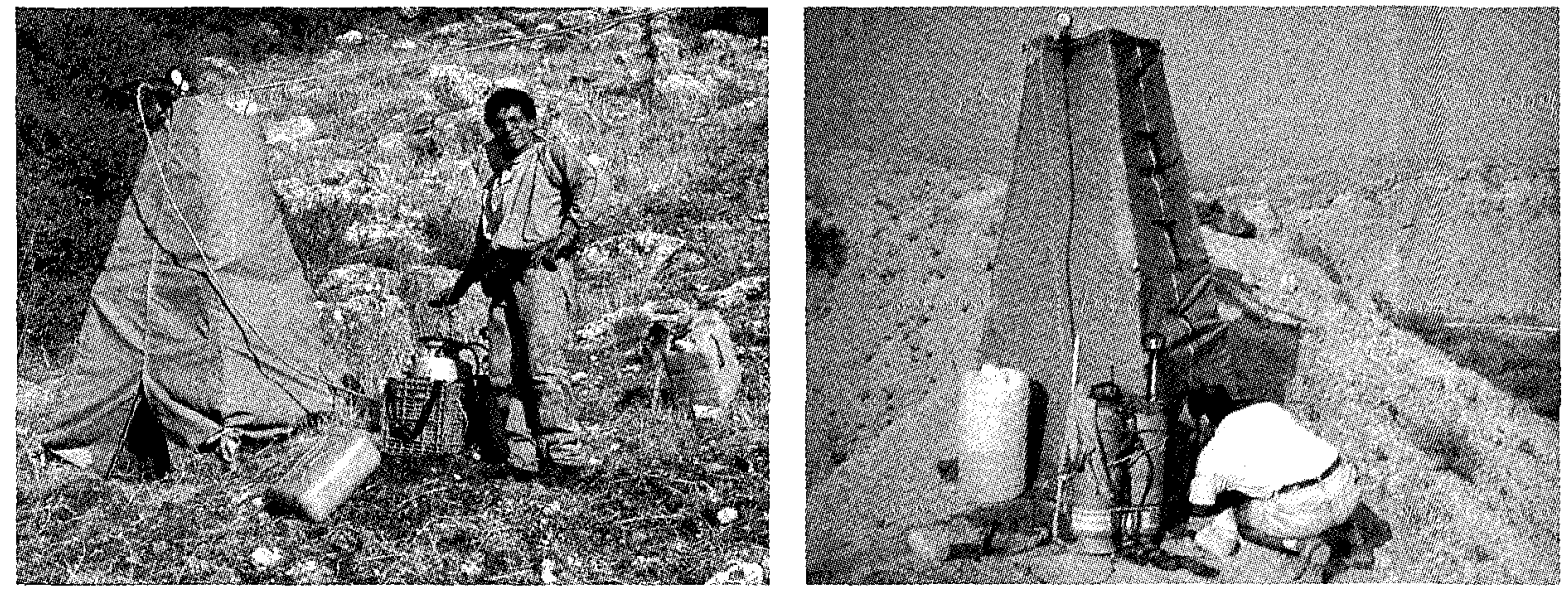

Figura 9. Simulador de lluvia pulverizador portátil (Cerdà et al., 1997): a) Israel y b) Almería. 


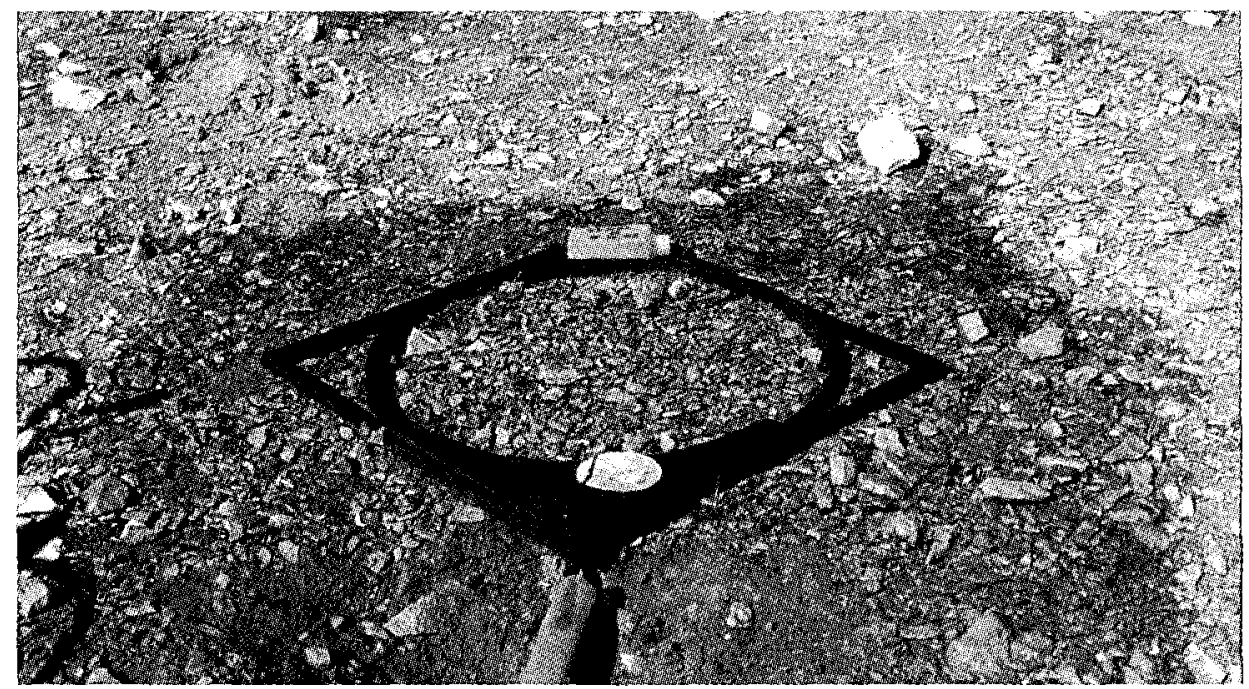

Figura 10. Parcela circular para lluvia simulada $\left(0,25 \mathrm{~m}^{2}\right)$ desarrollada en Israel para el simulador Cerdà et al., (1997). Diseño de Oren Ackermann.

pletamente nuevos, pero siempre manteniendo la transportabilidad y fácil montaje del simulador en el campo aunque las parcelas pueden ser muy grandes (Cerdà, 1995) (Figuras 11).

En algunas ocasiones, se ha preferido una automatización y sofisticación que ha llevado a la construcción de instrumentos de elevados costos (Neibling et al., 1981; Onstand et al., 1981; Herlihy et al., 1990; Hirschi et al., 1990). Estos diseños surgidos mayoritariamente en la década de los 80 están imbricados en la sexta generación, pero por sus características, se pueden clasificar dentro de la sépti-

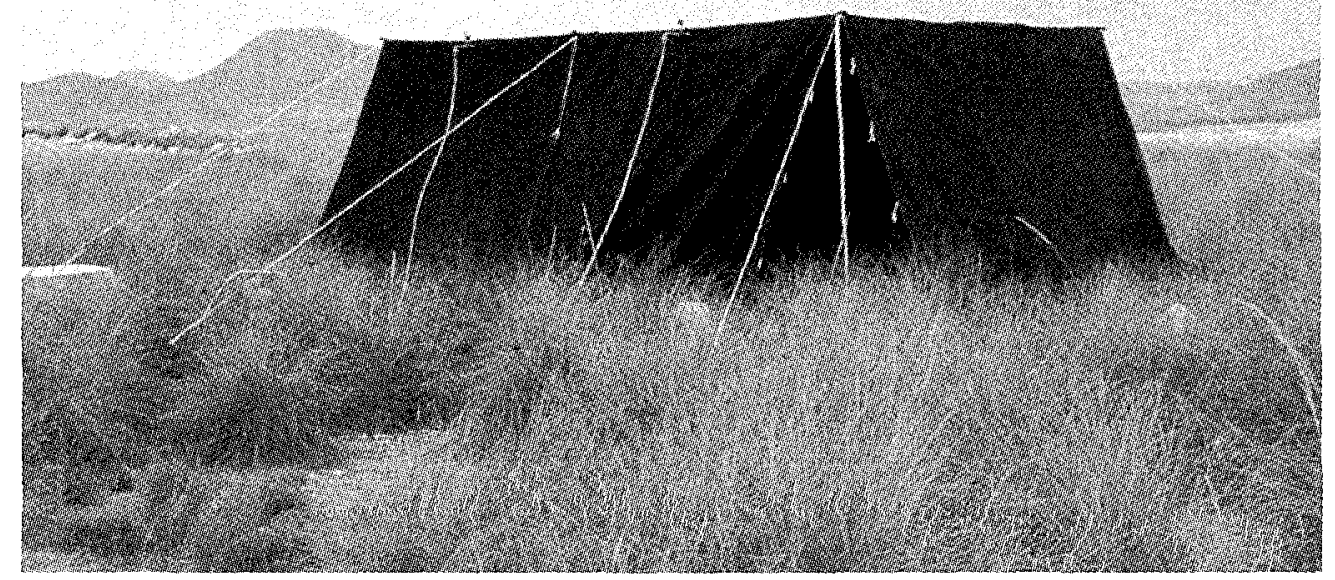

Figura 11. Simulador de lluvia de gran tamaño (parcelas de $5 \times 8 \mathrm{~m}$ ) desarrollado en Holanda (Cerdà, 1995). 
ma generación, en la cual la programabilidad de los simuladores es su mayor innovación (Bouyer-Bower y Burt, 1989).

Una de las mejoras con la sofisticación de estos simuladores de lluvia es la posibilidad de cambiar la intensidad de la lluvia durante la realización de los experimentos (Lusby, 1977; Marien y Vandewiele, 1986; Miller, 1987; Hirschi et al., 1990), o bien mecanizar el muestreo de la escorrentía, la filtración de ésta, etc., (Foster et al., 1982). La inconveniencia de la aparatosidad y reducida movilidad de estos instrumentos, hace que sean utilizados en laboratorio (Wilson y Rice, 1990), mientras que en algunos casos en los que se han superado los anteriores inconvenientes (Moore et al., 1983), van acompañados de equipos de medición automática, similares a los utilizados para las lluvias y escorrentías naturales (Essery et al., 1987).

Tabla 1. Principales referencias y características de los simuladores de lluvia. En esta lista se incluyen los modelos originales y aquellos que son fruto de importantes modificaciones. Autor (el primero de ellos), año, País, Tipo (A, goteador; B, pulverizador), características de formador (tipo de boquilla o material de los capilares), Tamaño de las gotas de lluvia $\left(\mathrm{D}_{50}\right.$, Diametro medio en relación al volumen, $\mathrm{mm})$, intensidades de la lluvia $\left(\mathrm{mm} \mathrm{h}^{-1}\right)$, tamaño de las parcelas utilizadas $(\mathrm{m})$ y tema estudiado (I, infiltración; E, erosión; R, escorrentía; S, erodibilidad; G, tamaño de gotas; $D$, drop-test; $N$, lavado de nutrientes; $P$, encharcamiento; $A$, salpicadura; T, lavado de pesticidas; L, lavado de sales; M, humedad del suelo; C, encostramiento). SS, Spraying System.

\begin{tabular}{|c|c|c|c|c|c|c|c|c|c|}
\hline & Autor & Año & Pais & Tipo & Formador & D50 & Intensidad & Parcelas & Uso \\
\hline 1 & Neal & 1938 & USA & B & & & $21-102$ & $1,2 \times 3,6$ & I \\
\hline 2 & Beutner & 1940 & USA & B & 1.5 Mulsifyre & & & $2 \times 8$ & I \\
\hline 3 & Rowe & 1940 & USA & A & Fibras & & & & I \\
\hline 4 & Laws & 1941 & USA & A & Bureta & $1-6$ & & & G \\
\hline 5 & Wilm & 1943 & USA & B & Type F & & $46-64$ & $2 \times 3,9$ & E-I \\
\hline 6 & McCalla & 1944 & USA & A & Bureta & $2,4-4,7$ & & & D \\
\hline 7 & Ellison & 1947 & USA & B & Fibras & & & & \\
\hline 8 & Dortignac & 1951 & USA & B & Type F & & 127 & $0,3 \times 0,8$ & E-I-R \\
\hline 9 & Vilenskii & 1953 & Rusia & A & Bureta & & & & D \\
\hline 10 & Low & 1954 & USA & A & Bureta & $5,5-5,75$ & & & D \\
\hline 11 & Rai & 1954 & USA & A & Bureta & & & & $\mathrm{D}$ \\
\hline 12 & Pereira & 1956 & USA & A & Bureta & & & & $\mathrm{D}$ \\
\hline 13 & Adams & 1957 & USA & A & Cp vidrio & 5,6 & 101 & $0,017 \mathrm{~m} 2$ & E-I-R \\
\hline 14 & Barnes & 1957 & USA & A & Hilos & & $25-152$ & $0,6 \times 0,6$ & I-R \\
\hline 15 & Parcker & 1957 & USA & B & Type F & & $25-152$ & $0,6 \times 1,8$ & E-I-R \\
\hline 16 & Meyer & 1958 & USA & B & SS 80100 & 2,1 & $64-127$ & $4 \times 11,5$ & $\mathrm{E}$ \\
\hline 17 & Rose & 1960 & Uganda & A & & $3,2-6,2$ & $50-152$ & & $\mathrm{E}$ \\
\hline
\end{tabular}


SIMULADORES DE LLUVIA Y SU APLICACIÓN A LA GEOMORFOLOGÍA. ESTADO DE LA CUESTIÓN

\begin{tabular}{|c|c|c|c|c|c|c|c|c|c|}
\hline 18 & Bertrand & 1961 & USA & $\mathrm{B}$ & $\mathrm{SECO} 7 \mathrm{LA} / 5 \mathrm{~B} / 5 \mathrm{D}$ & $0,6-1,2$ & 64-114 & $1,2 \times 1,2$ & $I$ \\
\hline 19 & McQueen & 1963 & USA & $\mathrm{A}$ & Cp de acero & 5,4 & 100 & 0,02 & $S$ \\
\hline 20 & Muchtler & 1963 & USA & A & Cp de acero & 3,2 & $38-50$ & $1,33 \mathrm{~m} 2$ & $\mathrm{E}$ \\
\hline 21 & Bubenzer & 1965 & USA & $\mathrm{B}$ & SS 80100 Veejet & 2,1 & 38 & $1 \times 5$ & E-R \\
\hline 22 & Chow & 1965 & USA & $A$ & Cp de polietileno & 3,2 & $19-33$ & $12 \times 12$ & $\mathrm{R}$ \\
\hline 23 & Hudson & 1965 & Rhodesia & $\mathrm{B}$ & Boquilla invertida & & & $1 \mathrm{~m} 2(\mathrm{x} 6)$ & $\mathrm{E}$ \\
\hline 24 & Hudson & 1965 & USA & $\mathrm{B}$ & SS 80100 & 2,1 & $64-127$ & $8,4 \mathrm{~m} 2$ & $\mathrm{E}$ \\
\hline 25 & Swanson & 1965 & USA & $\mathrm{B}$ & SS 80100 Veejet & 2,1 & $64-127$ & $4 \times 11$ & E-I \\
\hline 26 & Bisal & 1966 & USA & $\mathrm{A}$ & Fibras de lana & & & $0,0065 \mathrm{~m} 2$ & $\mathrm{~S}$ \\
\hline 27 & Epstein & 1966 & USA & A & Tubos de acero & 5,1 & $38-50$ & $1,33 \mathrm{~m} 2$ & $\mathrm{E}$ \\
\hline 28 & Kazó & 1966 & Hungria & $\mathrm{A}$ & & 4 & & $0,25 \mathrm{~m} 2$ & S \\
\hline 29 & Steinhardt & 1966 & Israel & $A$ & Tubos de acero & 2,8 & 6 & $0,5 \times 0,5$ & I \\
\hline 30 & Morin & 1967 & Israel & $\mathrm{B}$ & $\mathrm{AA} 1 \mathrm{HH} 12 / 1.5 \mathrm{H} 30$ & $1,9 / 2,6$ & $9-143$ & $1 \times 1,5$ & $\mathrm{E}$ \\
\hline 31 & Anderson & 1968 & USA & $\mathrm{B}$ & SS 80100 Veejet & 2,1 & $101-203$ & $5 \times 6,5$ & $\mathrm{E}$ \\
\hline 32 & Dixon & 1968 & USA & $\mathrm{B}$ & SECo 7LA & 1,2 & 119 & $1 \times 1$ & I-E \\
\hline 33 & Mazurak & 1968 & USA & $A$ & Cp de pl-stico & 5,1 & $20-120$ & $0,005 \mathrm{~m} 2$ & $\mathrm{E}$ \\
\hline 34 & Holland & 1969 & USA & $\mathrm{B}$ & Rainjet $78 \mathrm{C}$ & 1,4 & $36-58$ & $13 \times 26$ & E-I \\
\hline 35 & Lyles & 1969 & USA & $\mathrm{B}$ & SS 14WSQ/35WSQ & 2,1 & 18 & $1,5-31$ & $\mathrm{E}$ \\
\hline 36 & Meeuwing & 1969 & USA & $\mathrm{B}$ & Type F & & 127 & $0,5 \times 0,7$ & E-I \\
\hline 37 & Turner & 1969 & Australia & $\mathrm{B}$ & SS 8070 Veejet & & & $4,6 \times 4,6$ & $\mathrm{E}$ \\
\hline 38 & Amerman & 1970 & USA & $\mathrm{B}$ & Sprayco Nozzle $5 \mathrm{~d}$ & 1,2 & $1-150$ & $1,2 \mathrm{~m} 2$ & $\mathrm{~N}$ \\
\hline 39 & Black & 1970 & USA & A & Cp de polietileno & 3,2 & $19-33$ & $0,7 \times 0,7$ & $\mathrm{R}$ \\
\hline 40 & Bryan & 1970 & Canada & $B$ & & & & & $\mathrm{E}$ \\
\hline 41 & Costin & 1970 & Australia & $\mathrm{B}$ & Rose Sprayhead & 1,3 & 80 & $2 \times 3,3$ & E-I-N \\
\hline 42 & Chandra & 1970 & USA & B & Capilares & & & & $\mathrm{D}$ \\
\hline 43 & Schulz & 1970 & USA & $B$ & & & 28 & $3,7 \times 22,1$ & $\mathrm{E}$ \\
\hline 44 & Selby & $1970 \perp$ & Jueva Zelanda & $\mathrm{aA}$ & Alambres & & $20-300$ & $0,015 \mathrm{~m} 2$ & I \\
\hline 45 & Bubenzer & 1971 & USA & A & Cp de polietileno & $2,2-4,9$ & $10-525$ & $1,3 \times 1,3$ & E-P \\
\hline 46 & Cluff & 1971 & USA & $B$ & AA 1.5 H30 Fulljet & 2,6 & $17-1520$ & $1,5 \times 1,5$ & E-I \\
\hline 47 & Woolhiser & 1971 & USA & $\mathrm{B}$ & & & 35 & $0,3 \times 2,4$ & $\mathrm{~A}$ \\
\hline 48 & Black & 1972 & USA & $\mathrm{A}$ & Cp de polietileno & 3,2 & $19-33$ & $2 \times 2$ & $\mathrm{R}$ \\
\hline 49 & Rawitz & 1972 & Israel & $B$ & Sprayco Nozzle 5b & 1,2 & $3,7-100$ & $1 \times 2$ & I \\
\hline 50 & Karl & 1973 & Alemania & $B$ & Perrot & & $29,5-72,5$ & & $\mathrm{E}$ \\
\hline 51 & Blackburn & 1974 & USA & $\mathrm{A}$ & Tubos de acero & 2,6 & $5-250$ & $1 \times 1$ & E-I \\
\hline 52 & Bryan & 1974 & Canada & $\mathrm{B}$ & $1,1 \mathrm{~mm}$ & $0,39-0,79$ & $12,7-910$ & $0,51 \times 0,51$ & $\mathrm{~S}$ \\
\hline 53 & Kinell & 1974 & Australia & $\mathrm{A}$ & Agujas hipodérmicas & $3,8-5,1$ & $0-300$ & $1 \times 1$ & $\mathrm{E}$ \\
\hline 54 & Kleijn & 1974 & USA & $\mathrm{A}$ & Capilares & & & & $\mathrm{E}$ \\
\hline 55 & Lattanzi & 1974 & USA & $\mathrm{B}$ & SS 80100 Veejet & 2,1 & 64 & $0,61 \times 0,61$ & $\mathrm{E}$ \\
\hline 56 & Marsh & 1974 & USA & $B$ & $\mathrm{AA} 1 \mathrm{HH} 12 / 1.5 \mathrm{H} 30$ & $1,9 / 2,6$ & $9-143$ & $1 \times 1$ & $\mathrm{E}$ \\
\hline 57 & Brockman & 1975 & USA & $\mathrm{B}$ & SS7309/8015 Flat & & $17-282$ & & $\mathrm{~T}$ \\
\hline 58 & Bruce & 1975 & Nigeria & A & Glass tube & 6,7 & & & $S$ \\
\hline 59 & Gabriels & 1975 & Bélgica & A & Tubos de Cobre & & $4,7-64,5$ & & $\mathrm{E}$ \\
\hline 60 & Gierson & 1975 & Australia & $\mathrm{B}$ & SS $1.5 \mathrm{H} 30$ & 2,4 & $10-150$ & $1 \times 1$ & $E$ \\
\hline 61 & Nassif & 1975 & Reino Unido & $\mathrm{B}$ & Childs (PVC) & & $0-300$ & $4,1 \times 6,2$ & E-I \\
\hline 62 & Ponce & 1975 & USA & $B$ & Type F & & $25-127$ & $3,7 \mathrm{~m} 2$ & $\mathrm{E}$ \\
\hline 63 & Romkens & 1975 & USA & A & Agujas hipodérmicas & 2,7 & $12,2-104.1$ & $0.01 \mathrm{~m} 2$ & E-I \\
\hline 64 & Imeson & 1976 & Holanda & $\mathrm{A}$ & $5 \mathrm{~mm}$ tube & 5,75 & & & S \\
\hline
\end{tabular}




\begin{tabular}{|c|c|c|c|c|c|c|c|c|}
\hline 65 & Munn & 1976 & USA & A Tubos de polietileno & 3,2 & $31,2-250$ & $0,6 \times 0,6$ & S-I \\
\hline 66 & Yair & 1976 & Israel & Aspersores & & 2,6 & $200 \mathrm{~m} 2$ & $\mathrm{E}$ \\
\hline 67 & Ghadri & 1977 & USA & A & $2,8-6,7$ & & & S \\
\hline 68 & Grierson & 1977 & Australia & B AA 1HH12/ 1.5 H30 & 2,5 & 70 & $1 \times 1$ & $\mathrm{E}$ \\
\hline 69 & Imeson & 1977 & Holanda & Capilares & 3 & $40-120$ & $0,5 \mathrm{~m} 2$ & E-I \\
\hline 70 & Lusby & 1977 & USA & Rainjet $78 \mathrm{C}$ & 1,5 & 64 & $4 \times 22,1$ & E-I \\
\hline 71 & Peterson & 1977 & USA & A Tubos de Teflon & 3,6 & $76-203$ & $1,2 \times 1,2$ & $\mathrm{E}$ \\
\hline 72 & Riezebos & 1977 & Holanda & A & & & & $\mathrm{E}$ \\
\hline 73 & Savat & 1977 & Bélgica & A Capilares de silicona & 4,1 & 30 & $1 \times 0,2$ & $\mathrm{E}-\mathrm{A}$ \\
\hline 74 & Shriner & 1977 & USA & B Beta Fog SRN303 & 0,9 & $5-27$ & $1 \times 1$ & $\mathrm{I}-\mathrm{N}$ \\
\hline 75 & Walker & 1977 & Australia & A Agujas hipodérmicas & 3,2 & $20-250$ & $0,6 \times 3,0$ & E \\
\hline 76 & Walker & 1977 & USA & B & 2,77 & $30-140$ & $0,89 \times 0,58$ & S \\
\hline 77 & Asseline & 1978 & Camerun & SS 80100 Veejet & & $30-90$ & $1 \times 1$ & E-I \\
\hline 78 & Bryan & 1978 & Canada & B Aspersores oscilantes & 2,5 & $16-30$ & $15-50 \mathrm{~m} 2$ & $\mathrm{R}$ \\
\hline 79 & Chandra & 1978 & USA & A & & & & $\mathrm{D}$ \\
\hline 80 & Farres & 1978 & Reino Unido & A Capilares de Silicona & & 20 & & S \\
\hline 81 & Farres & 1978 & Reino Unido & Boquilla & & 120,7 & & $S$ \\
\hline 82 & Laflen & 1978 & USA & SS 80100 Veejet & 2,1 & 63,5 & $3,05 \times 10,67$ & E-T \\
\hline 83 & Malekuti & 1978 & USA & A Agujas hipodérmicas & 2,5 & $38-250$ & $0,6 \times 0,6$ & E-I-L \\
\hline 84 & Marston & 1978 & Australia & B AA 1.5 H30 Fulljet & 2,6 & $58-115$ & $1 \times 1,5$ & $\mathrm{E}$ \\
\hline 85 & McKay & 1978 & Australia & B SS 80100 Veejet & 2,2 & $30-200$ & $4 \times 22,5$ & E-I \\
\hline 86 & Ponce & 1978 & USA & Type F & & $25-127$ & $0,3 \times 0,77$ & $\mathrm{E}$ \\
\hline 87 & Brakensiek & 1979 & USA & A Agujas hipodérmicas & 3 & $5-102$ & $1 \times 2$ & I \\
\hline 88 & Bubenzer & 1979 & USA & B SS 14WSQ Fulljet & 1,7 & $1-50$ & $2 \times 2$ & I \\
\hline 89 & Foster & 1979 & USA & SS 80100 veejet & 2,25 & $31-135$ & $15 \times 20$ & $\mathrm{E}$ \\
\hline 90 & Grieve & 1979 & USA & A & 5,5 & & & $S$ \\
\hline 91 & Hamon & 1979 & USA & B & & & & E-I \\
\hline 92 & Meyer & 1979 & USA & SS $80100 / 80150$ & $1,6-2,5$ & $10-127$ & $0,7 \times 0,9$ & $\mathrm{E}$ \\
\hline 93 & Young & 1979 & USA & SS 80100 Veejet & 2,1 & $64-127$ & $4 \times 41,2$ & $\mathrm{E}$ \\
\hline 94 & Dunne & 1980 & USA & SS 80100 Veejet & 2 & 70 & $1,8 \times 4,9$ & $\mathrm{R}$ \\
\hline 95 & Dunne & 1980 & USA & SS 80100 Veejet & 2,7 & 132 & $1,8 \times 4,9$ & S \\
\hline 96 & Elwell & 1980 & Rhodesia & Nozzle $5 \mathrm{~d}$ & 1,03 & 53 & $1 \times 1$ & E \\
\hline 97 & Farres & 1980 & Reino Unido & A Capilar de silicona & $2,8-6,5$ & 1 & & $S$ \\
\hline 98 & Johnston & 1980 & Reino Unido & B & & $40-75$ & $6 \times 6$ & E \\
\hline 99 & McGhie & 1980 & USA & B & & & & $\mathrm{E}$ \\
\hline 100 & Shedden & 1980 & Reino Unido & B Agujas hipodérmicas & 1,29 & $3-400$ & $1 \times 1$ & $S$ \\
\hline 101 & Shedden & 1980 & Reino Unido & B Agujas hipodérmicas & 2,7 & $3-400$ & $1 \times 1,5$ & $S$ \\
\hline 102 & Bergsma & 1981 & Holanda & A & 5,5 & & & S \\
\hline 103 & Bork & 1981 & Alemania & B & 2,1 & $119-218$ & $1 \mathrm{~m} 2$ & I \\
\hline 104 & De Ploey & 1981 & Bélgica & A Capilares con malla & 2,5 & 27 & $0,35 \times 2$ & S-A \\
\hline 105 & Floyd & 1981 & Reino Unido & B V-jet nozzle 80100 & 0,9 & 27 & $6 \times 4$ & $\mathrm{E}$ \\
\hline 106 & KerËnyi & 1981 & Hungría & A & 4 & & $0,25 \mathrm{~m} 2$ & $S$ \\
\hline 107 & Niebling & 1981 & USA & SS 80100 Veejet & & $0-130$ & $5,32 \times 3,2$ & $\mathrm{E}$ \\
\hline 108 & Onstad & 1981 & USA & A Agujas hipodérmicas & 2,5 & $0-200$ & $0,91 \times 1,52$ & I \\
\hline 109 & Marston & 1982 & USA & B SS Fulljet 1 1/2 H30 & 2,6 & 112 & $1 \times 1$ & $\mathrm{E}$ \\
\hline 110 & Neff & 1982 & USA & Rain Jet $78 \mathrm{C}$ & 1,2 & 127 & $37 \mathrm{~m} 2$ & E-R \\
\hline 111 & Parcker & 1982 & USA & B Rainjet Brand N0. 78 & 2,75 & 66,3 & $9,1-15,2$ & $\mathrm{E}$ \\
\hline
\end{tabular}




\begin{tabular}{|c|c|c|c|c|c|c|c|c|}
\hline 112 & Scoging & 1982 & Reino Unido & B "Warley" Eclypse Ltd." & & 44 & $1 \mathrm{~m} 2$ & $\mathrm{I}-\mathrm{E}$ \\
\hline 113 & Zegelin & 1982 & USA & $\mathrm{B}$ & & & & $\mathrm{E}$ \\
\hline 114 & Epema & 1983 & Holanda & A & & & & G \\
\hline 115 & Loch & 1983 & Australia & SS 80100 Veejet & 2,1 & 100 & $3 \times 4$ & $\mathrm{E}$ \\
\hline 116 & Moeyersons & 1983 & Bélgica & A Capilares con malla & 2,5 & 50 & $0,23 \times 0,15$ & $\bar{S}$ \\
\hline 117 & Moore & 1983 & USA & SS Veejet 80150 & 2,5 & $10-100$ & $4,5 \times 22$ & $\mathrm{E}$ \\
\hline 118 & Schmidt & 1983 & Alemania & Capilares & 2,5 & $24-288$ & $2,5 \times 10$ & R-E-I \\
\hline 119 & Bryan & 1984 & Canada & Yoder sprinkler & & 29 & $28,4 \mathrm{~m} 2$ & $\mathrm{E}$ \\
\hline 120 & Sanroque & 1984 & España & A Agujas hipodérmicas & 2,7 & 100,7 & $0,4 \times 0,4$ & $S$ \\
\hline 121 & Bubenzer & 1985 & USA & $\mathrm{A}$ & & & $1 \mathrm{~m} 2$ & I \\
\hline 122 & Farres & 1985 & USA & A & $2,9-3,8$ & & & S \\
\hline 123 & Luk & 1985 & Canada & $\mathrm{B}$ & $0,4-5,8$ & 50,8 & $0,84 \mathrm{~m} 2(6)$ & $\mathrm{R}$ \\
\hline 124 & McKersie & 1985 & Reino Unido & A Spray Tec 40292630 & $0,72-1,95$ & $136-140$ & $1,96 \mathrm{~m} 2$ & $\mathrm{E}$ \\
\hline 125 & McKersie & 1985 & Reino Unido & SS $1 / 2 \mathrm{HH} 40$ & $1,35-2,38$ & $52,5-176$ & $3 \times 3$ & $\mathrm{I}$ \\
\hline 126 & Roth & 1985 & Alemania & Capilares & 2,89 & 60 & $0,5 \times 0,5$ & R-E \\
\hline 127 & Schultz & 1985 & USA & A Agujas hipodérmicas & 3 & 90 & $1 \times 0,18$ & $\mathrm{R}-\mathrm{E}$ \\
\hline 128 & Wood & 1985 & USA & B SS 0,635 G10 Fulljet & & 113,5 & $1 \times 1$ & I-E \\
\hline 129 & Auerswald & 1986 & Alemania & Veejet-80100 & & 63 & $4 \times 10$ & $\mathrm{E}$ \\
\hline 130 & Benito & 1986 & España & Matabi (super) & 2,2 & $15-75$ & $0,15 \mathrm{~m} 2$ & S \\
\hline 131 & Bradford & 1986 & USA & A Cp de polietileno & & & & $\mathrm{E}$ \\
\hline 132 & Luk & 1986 & Canada & B SPRACO Jet Nozzle & 2,4 & $77,4-144,8$ & $1,8 \times 5,5$ & E-R \\
\hline 133 & Nammah & 1986 & USA & B Aspersores de jardin & & 75 & $9,15 \times 0,3$ & $\mathrm{R}$ \\
\hline 134 & Pederson & 1986 & Australia & B SS $1.5 \mathrm{H} 30$ & & $20,6-100$ & $1 \times 1$ & E-I \\
\hline 135 & Wilcox & 1986 & USA & SSCo $1 / 4$ full jet & 1,2 & 100 & $1 \mathrm{~m} 2$ & E \\
\hline 136 & Egashira & 1987 & Japón & Agujas & & $33,5-66,1$ & $0,3 \times 0,1$ & S \\
\hline 137 & Farres & 1987 & Reino Unido & A Capilares de Silicona & & 40 & 0,005 & $S$ \\
\hline 138 & Hino & 1987 & Japón & A & & $20,2-29,9$ & $0,4 \times 1,5$ & I \\
\hline 139 & Jorgensen & 1987 & USA & Capilares & & 75 & $0,5 \mathrm{~m} 2$ & I \\
\hline 140 & Kamphorst & 1987 & Holanda & A Capilares de plástico & 5,9 & 360 & $0,0625 \mathrm{~m} 2$ & I-S \\
\hline 141 & Miller & 1987 & USA & SS 30WSQ & 2,5 & $12,7-115,8$ & $1 \times 3$ & E-S-I \\
\hline 142 & Norton & 1987 & USA & Tubos de acero & 4,6 & 63 & $0,14 \mathrm{~m} 2$ & $S$ \\
\hline 143 & Sanchez & 1987 & USA & SSCo $1 / 4-G 10$ & & 94,6 & $1 \mathrm{~m} 2$ & $\mathrm{I}$ \\
\hline 144 & Tossell & 1987 & Canada & SS HH-1/2 $30 \mathrm{~W}$ & 2,7 & $30-169$ & $1 \times 1$ & E-R \\
\hline 145 & Wilson & 1987 & USA & B & & 50 & $2,4 \times 9,8$ & E \\
\hline 146 & Calvo & 1988 & España & HARDI- $1553-10$ & & $34,5-80,2$ & $0,24 \mathrm{~m} 2$ & I-R-E \\
\hline 147 & Glanville & 1988 & Australia & 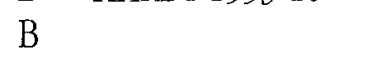 & & 72 & $0,01 \mathrm{~m} 2$ & $S$ \\
\hline 148 & Hassel & 1988 & Alemania & $\mathrm{B}$ & $2,36-2,65$ & $46,8-165,6$ & & E \\
\hline 149 & Johnson & 1988 & USA & $B$ & & 127 & $37 \mathrm{~m} 2$ & R-E \\
\hline 150 & Sutherland & 1988 & Canada & B & & 29 & $9 \mathrm{~m} 2$ & E \\
\hline 151 & Wolfgarten & 1988 & Alemania & Veejet-80100 & & $68,7-70,3$ & & $\mathrm{E}$ \\
\hline 152 & Beke & 1989 & Canada & $5,56-9,53 \mathrm{~mm}$ & & $11,7-31,7$ & $6 \times 12$ & $\mathrm{E}$ \\
\hline 153 & Ben-Hur & 1989 & Israel & B "Nelson" Koor-Shinitsk & & 100 & $1,5 \times 2$ & $\mathrm{R}$ \\
\hline 154 & Booth & 1989 & USA & $\mathrm{B}$ & & 200 & 1 & I \\
\hline 155 & Bowyer-Bower & 1989 & Reino Unido & B & $1,3-3,2$ & $10-120$ & $8 \times 8$ & E-R \\
\hline 156 & Bowyer-Bower & 1989 & Reino Unido & A Capilares de plástico & 2,3 & $10-120$ & $0,5 \mathrm{~m} 2$ & E-R \\
\hline 157 & Brandt & 1989 & Reino Unido & Bureta & & & & G \\
\hline 158 & Crouch & 1989 & Australia & $\mathrm{B}$ & & & & $\mathrm{E}$ \\
\hline
\end{tabular}




\begin{tabular}{|c|c|c|c|c|c|c|c|c|}
\hline 159 & De Lima & 1989 & USA & A Tubing-tip drop formes & er 3,5 & & & $\mathrm{D}$ \\
\hline 160 & DeBoer & 1989 & Canada & A & & $20-25$ & $0,6 \mathrm{~m} 2$ & $\mathrm{E}$ \\
\hline 161 & Halder & 1989 & Reino Unido & A Delavan 1/2 BQM 32 & 1,5 & 43 & $2,5 \times 5,5$ & $\mathrm{E}$ \\
\hline 162 & Hubbard & 1989 & USA & B SSCo Veejet nozzles & 0,6 & $43-125$ & $0,5 \times 1$ & $\mathrm{~N}$ \\
\hline 163 & Keren & 1989 & Israel & Capilares & 2,97 & 35 & $0,75 \times 0,60$ & I \\
\hline $164 \mathrm{I}$ & Bissonnais & 1989 & Francia & Capilares & 2,5 & 30 & $0,5 \times 0,5$ & $S$ \\
\hline 165 & Pelegrin & 1989 & España & Goteros & & 100 & 0,02 & $\mathrm{I}$ \\
\hline 166 & Francis & 1990 & Reino Unido & B SPRAYCO 1110191423 & 32,1 & $65,6-123,8$ & $1 \mathrm{~m} 2$ & $\mathrm{E}$ \\
\hline 167 & Francis & 1990 & Reino Unido & TOPLAS & 0,9 & $18,3-31,5$ & $2 \times 5$ & E \\
\hline 168 & Herlihy & 1990 & USA & Sprayjet WSQ30 & $1,7-2,3$ & 60 & $4 \mathrm{~m} 2$ & $\mathrm{E}$ \\
\hline 169 & Hinkle & 1990 & USA & B SS Fulljet HH30WSQ & 6 & 100 & $40 \mathrm{~m} 2$ & $\mathrm{E}$ \\
\hline 170 & Hirschi & 1990 & USA & B SS Veejet 80100 & 2 & $2,5-116$ & $4,5 \times 22,1$ & $\mathrm{E}$ \\
\hline 171 & Kinell & 1990 & Australia & Capilares & 2,7 & 64,7 & $0,8 \times 0,8$ & $\mathrm{E}$ \\
\hline 172 & Mulligen & 1990 & Holanda & B SSCo Tee Jet 20W & & 70 & $2 \times 10$ & $\mathrm{I}-\mathrm{R}$ \\
\hline 173 & Navas & 1990 & España & B Fulljet $1 / 8$ GC6SQ & $0,93-1,49$ & $48-58$ & $1,25 \times 1,25$ & $\mathrm{E}-\mathrm{L}$ \\
\hline 174 & Parsons & 1990 & Reino Unido & B LECHLER 483.427 & 1,7 & 80 & $18 \times 35$ & E-R \\
\hline 175 & Poesen & 1990 & Bélgica & Lechler, 460788 & 2 & 36,4 & $0,57 \mathrm{~m} 2$ & $S$ \\
\hline 176 & Richter & 1990 & Alemania & A & & & & $\mathrm{E}$ \\
\hline 177 & Smith & 1990 & Israel & A Agujas hipodérmicas & 2,97 & 33 & $0,6 \times 0,75$ & $\mathrm{I}-\mathrm{E}$ \\
\hline 178 & Tossell & 1990 & Canada & B SS $1 / 84.3 \mathrm{~W}, 1 / 230 \mathrm{~W}$ & 2,7 & $16-350$ & $1 \times 1$ & E-R \\
\hline 179 & Uresti Gil & 1990 & Reino Unido & A Delavan 1/2 BQM 32 & $0,9-1,86$ & $11,7-133$ & $3 \times 3$ & $\mathrm{I}$ \\
\hline 180 & Gil & 1990 & Reino Unido & SSCo $1 / 2 \mathrm{HH} 25$ & $0,88-0,98$ & $23,5-47,1$ & $3 \times 3$ & I \\
\hline 181 & Cervera & 1991 & España & B & $1,53-2,97$ & $20-130$ & $0,5 \times 1,5$ & $\mathrm{E}-\mathrm{R}$ \\
\hline 182 & DeBoer & 1991 & USA & Aspersores & & 115 & $5,5 \times 25$ & $\mathrm{E}$ \\
\hline 183 & Gerits & 1991 & Holanda & Capilares & $4-5$ & 40 & $0,5-0,78 \mathrm{~m} 2$ & I-R-E \\
\hline 184 & Gerits & 1991 & Holanda & B & Fog-type & $35-52$ & $75-100$ & E-R \\
\hline 185 & Hofmann & 1991 & USA & Rainjet $78 \mathrm{C}$ & 1,5 & 46 & $4 \times 22,1$ & E-R-I \\
\hline 186 & Kainz & 1991 & Alemania & Veejet-80100 & & 66,3 & & $\mathrm{E}$ \\
\hline 187 & Ortiz & 1991 & España & B Aspersores de riego & & $5,5-8,8$ & $60 \times 60$ & E-I \\
\hline 188 & Wright & 1991 & Reino Unido & B SS $1 / 2 \mathrm{HH} 40$ & $1,6-2,33$ & $48-76$ & $3 \times 6$ & $\mathrm{E}$ \\
\hline 189 & Casenave & 1992 & Niger & B & & $30-150$ & & $\mathrm{R}$ \\
\hline 190 & Edwards & 1992 & Canada & A Tubos & 3,2 & $12,5-100$ & $0,914 \times 0,305$ & S \\
\hline 191 & Greene & 1992 & Australia & B AA $1 \mathrm{HH} 12 / 1.5 \mathrm{H} 30$ & $1,9 / 2,6$ & $9-143$ & 1 & E-R \\
\hline 192 & Hodges & 1992 & Canada & Yoder sprinkler & & 29 & $10,6-36,8$ & $\mathrm{~F}$ \\
\hline 193 & Melville & 1992 & Reino Unido & A SSCO $1 / 2$ HH 25 & 1,4 & 40 & $6 \times 3$ & E \\
\hline 194 & Wright & 1992 & Reino Unido & A SSCo $1 / 2$ HH 40 & $1,6-2,26$ & $48.2-76$ & $6 \times 3$ & $\mathrm{E}$ \\
\hline 195 & Huang & 1993 & USA & B SS Veejet 80150 & 2,5 & $50-90$ & $1,2 \times 1,2$ & $\mathrm{C}$ \\
\hline 196 & Medalus & 1993 & Reino Unido & A Lechler $402.648 / 460.72$ & & $30-80$ & $3 \times 4$ & $\mathrm{E}$ \\
\hline 197 & Nishimura & 1993 & Japon & A agujas & 2 & 31,5 & $0,3 \times 0,5$ & $\mathrm{C}$ \\
\hline 198 & Poesen & 1993 & Bélgica & B Lechler $461008-460788$ & 2,2 & $36-71$ & $1 \mathrm{~m} 2$ & E-R \\
\hline 199 & Teck & 1993 & Reino Unido & A Delavan 3/8 Bam 20 & $1,61-1,91$ & $122-141$ & $2 \times 2$ & S \\
\hline 200 & Bradford & 1994 & USA & B SSCo Veejet 10150 & & 70 & & $\mathrm{E}$ \\
\hline 201 & Cazzufi & 1994 & Italia & B & $1,8-4$ & 1 & $1 \times 1,5$ & $\mathrm{E}$ \\
\hline 202 & Evans & 1994 & Australia & $\mathrm{B}$ & & 100 & $1 \times 1$ & $\mathrm{E}$ \\
\hline 203 & Kamalu & 1994 & Reino Unido & B & 2 & $90-110$ & $1 \times 2,5$ & $\mathrm{E}$ \\
\hline 204 & Kapenzi & 1994 & Reino Unido & A Lechler $402768 / 30$ & 1,92 & $48-62$ & $3 \times 3$ & E-A \\
\hline 205 & Kerényi & 1994 & Hungria & $\mathrm{A}$ & & 30 & $0,56 \times 0,36$ & $\mathrm{E}$ \\
\hline
\end{tabular}


SIMULADORES DE LLUVIA Y SU APLICACIÓN A LA GEOMORFOLOGÍA. ESTADO DE LA CUESTIÓN

\begin{tabular}{|c|c|c|c|c|c|c|c|c|}
\hline 206 & Parsons & 1994 & Reino Unido & A Lechler $483.427 / 460$ & $8481,56 \sim 3,2$ & $40-120$ & $25 \mathrm{~m} 2$ & $E$ \\
\hline 207 & Sardo & 1994 & Italia & Dan 2200 & $1-2,5$ & $12-48$ & $2,5 \times 22$ & $\mathrm{E}$ \\
\hline 208 & Slattery & 1994 & Canada & B SPRAY 45900830BK & 1,3 & 32 & $0,81 \times 5$ & $\mathbf{E}$ \\
\hline 209 & Ternan & 1994 & Reino Unido & B & 0,58 & 90 & & $\mathrm{E}$ \\
\hline 210 & Cerdá & 1995 & Holanda & B SS $3 / 844 \mathrm{~S} 27 \mathrm{~W}$ & & 43 & $8 \times 5$ & E-S \\
\hline 211 & Hignett & 1995 & Australia & A Agujas hipodérmicas & $2,7-5,1$ & $40-70$ & $1,14 \times 1,4$ & $\mathrm{I}-\mathrm{S}$ \\
\hline 212 & Williams & 1995 & Reino Unido & A Solid cone brass & & 40 & $2 \mathrm{~m} 2$ & E-I \\
\hline 213 & Byars & 1996 & USA & B & & $30,5-127$ & $0,91 \times 1,83$ & $I$ \\
\hline 214 & Calder & 1966 & Reino Unido & $B$ & $0,6-5,2$ & $36-45$ & & $G$ \\
\hline 215 & Wan & 1996 & USA & A Agujas hipodérmicas & 2 & 65 & $0,76 \times 0,76$ & A \\
\hline 216 & Ben-Hur & 1997 & Istael & A Agujas hipodérmicas & 3 & 40 & $1 \times 1,3$ & $\mathrm{I}-\mathrm{S}$ \\
\hline 217 & Cerdá & 1997 & España & B HARDI- $1553-10$ & 2,49 & 54,6 & $0,24 \mathrm{~m} 2$ & E-R \\
\hline 218 & Levy & 1997 & Israel & Capilares & 2,97 & $30-33$ & $0,60 \times 0,75$ & $S$ \\
\hline 219 & Ogden & 1997 & USA & Capilares & & $9-36$ & $0,15 \times 0,4$ & $\mathrm{I}$ \\
\hline 220 & Rodriguez & 1997 & Venezuela & B AA $1 \mathrm{HH} 12 / 1.5 \mathrm{H} 30$ & 2,6 & 56 & $1 \times 5$ & $\mathrm{E}$ \\
\hline 221 & Erpul & 1998 & Bélgica & $\mathrm{B}$ & 1,1 & 37,7 & 143 & G \\
\hline 222 & Green & 1998 & USA & Capilares & & 125 & $0,5 \times 0,5$ & $\mathrm{E}$ \\
\hline 223 & Kranz & 1998 & USA & Capilares & & 33 & $1,2 \mathrm{~m} 2$ & $\mathrm{~T}-\mathrm{I}$ \\
\hline 224 & Oostwoud & 1998 & Alemania & B Lechler 461.008 .30 & $0,5-6$ & $47-102$ & $2,75 \times 12$ & $\mathrm{E}$ \\
\hline 225 & Perez & 1998 & USA & $5 \mathrm{~mm}$ & & 45 & $0,5 \times 0,5$ & M \\
\hline 226 & Rhoton & 1998 & USA & SS $80100 / 80150$ & & 64 & $0,6 \times 0,6$ & $\mathrm{E}$ \\
\hline 227 & Simón & 1998 & España & Plastic tube & 4 & 65 & $0,5 \times 0,5$ & $\mathrm{I}$ \\
\hline 228 & Geedes & 1999 & Australia & A $5 \mathrm{~mm}$ plastic tube & 5,7 & & & A \\
\hline 229 & CEBAS & - & España & $\begin{array}{l}\text { SS 3/820GGW- } \\
1 / 2 \mathrm{HH} 35-50 \mathrm{~W}\end{array}$ & $0,8-1,2-1,4$ & $33-55-84$ & $2 \times 2$ & $\mathrm{E}$ \\
\hline
\end{tabular}

\section{Ventajas e inconvenientes de los simuladores de lluvia}

La utilización de los simuladores de lluvia está circunscrita a la ventaja que supone el prescindir de la lluvia natural. Cuatro son las razones para esto: la lluvia simulada es más rápida de producir, más eficiente, más controlada y más adaptable que la lluvia natural (Meyer, 1985).

Respecto a los estudios con lluvia natural, se tiene que tener en cuenta que con la lluvia simulada se reduce el tiempo de experimentación, y con ello, el coste de la investigación. Además, se tiene el máximo control sobre el momento de la medición: estado previo de la parcela, características de la lluvia, evolución posterior, respuesta del suelo, etc.

En algunos casos, los simuladores de lluvia son totalmente insustituibles, debido al poco tiempo destinado a la investigación y a la rapidez e irregularidad de los procesos, además de los resultados poco satisfactorios de las metodologías tradicionales. En climas como el mediterráneo, donde la escasez de eventos de lluvia podría retrasar la investigación durante años, se hace totalmente necesario el 


\section{A. CERdà}

uso de esta técnica. La simulaciones de lluvia permiten tener acceso a una base de datos de gran calidad en un tiempo relativamente corto.

La simulación de lluvia, a pesar de ser una técnica muy alabada, también tiene sus inconvenientes. Se acusa a los simuladores de lluvia de ser poco representativos por ocupar un área muy reducida y no reproducir la lluvia natural con exactitud, además de tener en algunos casos costes elevados en diseño y mantenimiento.

El tamaño de la parcela es realmente una desventaja si se estudian los procesos a escala de cuenca, pero tanto si se usan a escala de micro-cuencas, de laderas o parte de éstas, de campos de cultivo, como si se usan a escalas menores a la métrica, la técnica es totalmente factible, y diríamos incluso imprescindible en lo relativo a superficies de tamaño reducido.

Por lo que respecta a la no reproducción de la lluvia natural, esto se puede entender como una imposibilidad, o bien como una pérdida de tiempo el intentarlo, ya que en el caso de necesitar exactamente la misma lluvia simulada que la natural, no sería necesario el simularla, sino tan sólo esperar a que ésta se produjera. Además, los estudios con lluvia simulada no pretenden sustituir las investigaciones realizadas con lluvia natural, sino complementarlas. En el caso de trabajos acerca de la respuesta de los ecosistemas ante precipitaciones de larga recurrencia, sólo pueden ser investigados con lluvia simulada, ya que lluvias de diez, veinte o cincuenta años de periodo de retorno son difícilmente estudiadas con otros métodos por el largo periodo de tiempo necesario.

La existencia de múltiples tipos de lluvia a imitar y su variabilidad hace que lo más coherente sea poder repetir el mismo experimento tantas veces como se quiera, y que el diseño de éste (intensidad, duración, etc., de la lluvia), puede ser modificado por el investigador. Para gran parte de los investigadores la conclusión final es que cuando el tema objeto de estudio está de acuerdo con los presupuestos científicos requeridos por la técnica de la simulación de lluvia, los inconvenientes son insignificantes respecto a las ventajas.

\section{La expansión geográfica de los simuladores de lluvia.}

Los simuladores de lluvia existen en América del Norte desde finales de los años treinta, aunque pronto aparecieron en algunos países africanos de habla inglesa, fruto de la influencia del profesor Hudson (1964; 1965). En Europa, aunque existieron con anterioridad, fue en los años 70 cuando empezaron a ser abundantes, especialmente en Inglaterra, Holanda, Bélgica y Alemania.

La fuerte expansión de los simuladores de lluvia en las dos últimas décadas llega hasta los países Sudamericanos, la India (influencia británica), Japón, y ya en los años ochenta a los países de la cuenca mediterránea. Entre estos últimos, Israel es una excepción, tanto por la influencia americana, como por el desarrollo de simuladores de lluvia propios (Morin et al., 1967). 
Los simuladores de lluvia en España aparecieron en la década de los 80, por lo que ésta es una técnica muy reciente en nuestro país, sobre todo si lo comparamos con los países de habla anglosajona.

Este instrumento ha sido bien recibido entre los investigadores españoles debido a que sus características (costes bajos en relación con otras técnicas, y gran rapidez en la obtención de los datos), se adecuan perfectamente a la problemática del investigador nacional. La escasez de recursos económicos en algunos grupos de investigación y la falta de datos han sido el caldo de cultivo que ha hecho que los simuladores de lluvia se desarrollaron con profusión en los últimos quince años.

Hasta el momento en el estado Español, se conocen ocho modelos de simuladores de lluvia por medio de la bibliografía. Cuatro de estos aparatos están destinados a experimentos de campo (Calvo et al., 1988; Navas et al., 1990; Cerdà et al., 1997) pero que se podrían utilizar en el laboratorio; dos de uso exclusivo en el campo (Pelegrin, 1989; Ortiz, 1991) y otros dos en el laboratorio (Sanroque et al., 1984; Benito et al., 1986). En la actualidad, el CEBAS-CSIC de Murcia está desarrollando un modelo para uso en parcelas de $4 \mathrm{~m}^{2}$ con intensidades de lluvia entre 33 y $84 \mathrm{~mm} \mathrm{~h}^{-1}$ (Com. Per. M.M.Mena, V. Castillo y J. Albadalejo).

Algunos grupos de investigadores han introducido pequeñas pero importantes mejoras en los anteriores simuladores. En general han conseguido instrumentos más adecuados a sus necesidades al modificar la forma de la parcela (De Alba, 1997), mejorar la transportabilidad del equipo, o perfeccionar el sistema de presurización (Ruiz-Flaño, 1993; Arnáez Vadillo et al., 1996).

Tabla 2. Simuladores de lluvia desarrollados por países.

\begin{tabular}{|c|c|c|c|c|c|}
\hline País & $\mathbf{n}^{\mathbf{o}}$ & País & $\mathbf{n}^{\mathbf{o}}$ & País & $\mathbf{n}^{\mathbf{0}}$ \\
\hline USA & 102 & Israel & 9 & Francia & 1 \\
\hline Reino Unido & 32 & Bélgica & 7 & Niger & 1 \\
\hline Australia & 17 & Italia & 3 & Nigeria & 1 \\
\hline Canadá & 14 & Hungria & 2 & Nueva Zelanda & 1 \\
\hline Alemania & 10 & Japón & 2 & Rusia & 1 \\
\hline Holanda & 10 & Rhodesia & 2 & Uganda & 1 \\
\hline España & 9 & Camerún & 1 & Venezuela & 1 \\
\hline
\end{tabular}

\section{Conclusiones}

La revisión bibliográfica realizada ha permitido constatar la gran pujanza de la simulación de lluvia en el seno de la geomorfología. No es una técnica ideada y desarrollada por los geomorfólogos, pero si ha sido modificada y acondicionada 
para el tipo de estudios que desarrolla nuestro colectivo. Los simuladores de lluvia de laboratorio, principalmente los desarrollados a partir de goteadores, son los menos utilizados por su aparatosidad. En cambio, los geomorfólogos han recibido con gran entusiasmo los simuladores pulverizadores de reducido tamaño por su reducido coste y su uso en estudios de campo.

\section{Referencias bibliogräficas}

ADAMS, J.E., KirKham, D. y NIELSEN, D.R. (1957): A portable rainfall-simulator infiltrometer and physical measurements of soil in place. Soil Sci. Soc. Am. Proc., 21, 473-477.

Amerman, C.R., Hillel, D.I. y Peterson, A.E. (1970): A variable-intensity sprinkling infiltrometer. Soil Sci. Soc. Am. Proc., 34, 830-832.

ANDERSON, J.U. STEWART, A.E. Y GREGORY, P.C. (1968): A portable rainfal simulator and runoff sampler. New Mexico State Univ. Ag. Espt. Sta. Rept 143, Las Cruces, 5 p.

arnáez Vadillo, J., Ruiz Flaño, P. y Lasanta Mar'í́nez, T. (1996): Comportamiento hidromorfológico de los microambientes de campos abandonados con lluvias intensas: experiencias en el valle de Aisa (Pirineo Aragonés). En Grandal D., Anglade, A. y Pagés Valcarlos, J. (Eds.), IV Reunión de Geomorfología. SEG. 659-669.

Asseline, J. y Valentin, C. (1978): Construction et mise au point d'un imfiltromètre à aspersion. Cabiers ORSTOM, série Hydrologie, XV (4): 321-349.

Auerswald, K. (1986): A Swanson-Type Rainfall Simulator for Erosion Studies. Mitteilgn. Dtsch. Bodenkundl. Gesllsch., 49, 224-225.

Barnes, O.F. y Costel, G. (1957): A mobile infiltrometer. Agronomy Journal, 49, 105-107.

BECHER, H.H. (1990): Comparison of German and Swiss rainfall simulatorsRelative srop energies and their distribution in time and space for simulates rains. Z. Pflanzenernabr. Bodenk., 153, 409-14.

Beke, G.J., Foroud, N., Channappa, T.C. y Entz, T. (1991): Runoff and Soil Loss from Experimental Plots in Southern Alberta Using Simulated Rainfall. Canadian Agricultural Engineering, 205-210.

Ben-Hur, M. y AGassI, M. (1997): Predicting interrill erodibility factor from measured infiltration rate. Water Resources Research, 33, 2049-2415.

Ben-Hur, M. y LetEY, J. (1989): Effect of Polysaccharides, Clay Dispersion, and Impact energy on Water Infiltration. Soil Sci. Soc. Am. J., 53 (1) 233-238.

Benito-Rueda, E., Gómez-Ulla, A. y Díaz-Fierros Viqueira, F. (1986): Descripción de un Simulador de Lluvia para estudios de erodibilidad del Suelo y Estabilidad de los Agregados al agua. An. Edafol. Agrobiol., XLV (9-10) 1115-1126. 
SIMULADORES DE LLUVIA Y SU APLICACIÓN A LA GEOMORFOLOGÍA. ESTADO DE LA CUESTIÓN

Bergsma, E. y VAlenzuela, C.R. (1981): Drop testing aggregate stability of some soils near Merida, Spain. Earth Surface Processes and Landforms, 6, 309-318.

Bertrand, A.R. y PARR, J.F. (1961): Desing and operation of the Pardue sprinkling infiltrometer. Pardue Univ. Res. Bull. No. 723.

Beutner, E.L., GaEBe, R.R. y Horton, R.E. (1940): Sprinkled-plot runoff and infiltration experiments on Arizona desert soils. Trans. Am. Geophys. Union, 21, 550-558.

Bisal, F (1967): Infiltration Rate as Related to Rainfall Energy. Can.J. Soil Sci., 47, 33-42.

BLACK, P.E.(1970): Runoff from watershed models. Water Resources Research, 6, 465-477.

BLACK, P.E.(1972): Hydrograph responses to geomorphic model watershed characteristics and precipitation variables, Journal of Hydrology. 17, 309-329.

Blackburn, W.H., MeEuwing, R.O. y SkaU, C.M. (1974): A mobile infiltrometer for use on rangeland. Journal of Range Management, 27 (4) 322-323.

Booth, C.J. y PRICE, B.C. (1989): Infiltration, Soil Moisture, and Related Measurements at a Landfill with a Fractured Cover, Illinois. Journal of Hydrology, 108, 175-188.

BORK, H.-R. (1981): Oberflächenabfluss und infiltration. Ergebnisse von starkregensimulationene in der Süheide (ostniederdachwsen) und in Süost- Spanien. Deutscher Geographentag, 43, 159-163.

BOUYER-BOwer, T.A.S. y BuRT, T.P. (1989): Rainfall Simulators for Investigating Soil Response to Rainfall. Soil Technology, 2, 1-16.

Bowman, B.T., Brunke, R.R., Reynolds, W.D. y WALL, G.J. (1994): Rainfall simulator-grid system for solute transport studies using large, intact soil blocks. Journal of Environmental Quality, 23, 815-822.

Bradford, J.M. Remley, P.A. FerRis, J.E. y SANTINI, J.B. (1986): Effect of soil surface Sealing on splash from a single waterdrop. Soil Sci. Soc. Am. J. 50; 1547-52.

BRADFORD, J.M. y HUANG, CH. (1994): Interrill soil erosion as affected by tillage and residue cover. Soil and Tillage Research, 31, 353-361.

Brakensiek, P.O., RaWls, W.J. y Hamon, W.R. (1979): Application of an infiltrometer system for describing infiltration into soils. Transactions of the ASAE, 22 (1), 320-325.

BRANDT, C.J. (1989): The size distribution of throughall drops under vegetation canopies. Catena, 16, 507-524.

Brockman, F. E. DukE. W.B. y Hunt. J.K. (1975): A rainfall simulator for pesticide leaching studies. Weed Sci, 23 (6), 533-535.

Bruce-OKine, E. y LAL, R. (1975): Soil erodibility as determined by raindrop technique. Soil Sci., 119 (2) 149-57.

BRYAN, R.B. (1970): An improved rainfall simulator for use in erosion research. Canadian Journal of Earth Science, 1552-1561. 
Bryan, R.B. (1974): A Simulated Rainfall Test for the Prediction of Soil Erodibility. $Z$ Geomorph. N.F., 21, 138-150.

Bryan, R.B., Imeson, A.C. y CAMPBell, I.A. (1984): Solute realease and sediment entrainment on microcatchments in the Dinosaur Park Badlands Alberta, Canada. Journal of Hydrology, 71, 79-106.

Bryan, R.B., YAIR, A. y Hodges, W.K. (1978): Factor controlling the initiation of runoff and piping in Dinosaur Provincial Park badlands, Alberta, Canada. $Z$. Geomorph. N.F., Suppl. Bd., 29, 151-168.

Bubenzer, G.D. y Jones, B.A. (1971): Drop size and impact velocity effects on the detachment of soils under simulated rainfall. Transactions of the ASAE, 14 (4) 625-628.

BubenZER, G.D. y MEYer, L.D. (1965): Simulation of rainfall and soils for laboratory research. Transactions of the ASAE, 8, 73-75.

Bubenzer, G.D., Molnau, M. y McCool, D.K. (1979): Paulose rainfall simulator. Proceedings of the rainfall simulator workshop, USDA-SEA, ARS-W-10/july 1979, 131-133.

Bubenzer, G.D., Molnau, M. y McCool, D.K. (1985): Low intensity rainfall with a rotating disk simulator. Transactions of the ASAE, 28: 35-43.

Byars, B.W., ALLEN, P.M. y Bingham, N.L. (1996): A portable rainfall simulator for assessing infiltration. Journal of Soil and Water Conservation, 51, 508-510.

Calder, I.R. Hall, R.L., Rosier, P.T.W., Bastable, H.G., y Prasanna, K.T. (1996): Dependence of rainfall interception on drop size. 2. Experimental determination of teh wetting functions and two-layer stochastic model parameters fro five tropical tree species. Journal of Hydrology, 185, 379-388.

Calvo, A., Gisbert, B., Palau, E. y Romero, M. (1988): Un simulador de lluvia portátil de fácil construcción. En M. Sala y F. Gallart (Eds.): Métodos y técnicas para la medición de procesos geomorfológicos, S. E. G. Monografía 1, pp. 6-15

CARTER, C.E. Greer, J.D. y Floyd, J.M. (1974): Raindrop Characteristics in South Central United States. Transactions of the ASAE, 17 (6) 1033-1037 p.

Casenave, A. y Valentin, C. (1992): A runoff capability classification system bases on surface features criteria in semiárid areas of West Africa. Journal of Hydrology, 130, 231-249.

Cazzuffi, D., Monferino, F., Monti, R. y Rimoldi, P. (1994): Experimental evaluation of the erosion on bare and geosynthetically protected slopes. En Rickson, R.J. (De.): Conserving Soils Resources. European Perspectives, 413-421. CAB International. Cambridge

CERDÀ, A. (1995): Soil moisture regime under simulated rainfall in a three years abandonedfield in Southeast Spain. Physics and Chemistry of the Earth, 20 (34) $271-279$.

CERDÃ, A. (1998): Soil aggregate stability under different Mediterranean vegetation types. Catena, 32, 73-86. 
SIMULADORES DE LLUVIA Y SU APLICACIÓN A LA GEOMORFOLOGÍA. ESTADO DE LA CUESTIÓN

Cerdà, A., Ibáñez, S. y Calvo, A. (1997): Design and operation of a small and portable rainfall simulator for rugged terrain. Soil Technology, 11 (2) 161-168.

Cervera, M., Clotet, N., Guardia, R. y Sole, Ll. (1991): Response to rainfall simultation rrom scarcely vegetated and non-vegetated Badlands. Catena, 19, 39-56.

ChANDRA, S. y DE, S.K. (1970): A simple laboratory apparatus to measure relative erodiblity of soils. Soil Science, 25, 115-121

CHOw, V.T. y Yen, B.C. (1974): A laboratory watershed experimentation system. Hydr. Engr. Series no 27, Civil Engineering Dept., Univ. of Illinois, 196 p.

Chow, W.T. y Harbaugh, T.E. (1965): Raindrop production for laboratory watershed experimentation. J. Geophys. Res., 70 (24) 6111-6119.

CHRISTIANSEN, J.E. (1941): The uniformity of application of water by sprinkler system. Agricultural Engineering, 22, 89-92.

ClufF, C.B. (1971): The use of a realistic rainfall simulator to determine relative infiltration rates of contributing watershed to the Lower Gila below Point Rock Dam. Univ. of Arizona, Water Res Center. Tucson.

Costin, A.B. y Gilmour, D.A. (1970): Portable rainfall simulator and plot unit for use in fields studies of infiltration, runoff, and erosion. J. Appl. Ecol., 7 (1) 193200.

Crouch, R.J. y Colunson, J.E. (1989): An Air Injected, Single Nozzle Rainfall Simulator, Dewsigned for Use on Gully Sides. Australian Journal of Soil and Water Conservation, 2 (3) 37-39.

DE AlBA, S. (1997): Metodología para el estudio de la erosión en parcelas experimentales: relaciones erosión-desertificación a escala de detalle. En J.J. Ibáñez, B.L. Valero Garcés, y Machado, C. (Eds.): El paisaje mediterráneo a través del espacio y del tiempo. Implicaciones en la desertificación. Geoforma Ediciones, Logroño, 259-293.

De Boer, D.H. y CAMPBell, I.A. (1989): Spatial scale dependance of sediment dynamics in a semi-arid Badland drainage basin. Catena, 16, 277-90.

DE LiMA, J.L.M.P. (1989): Overland flow under rainfall: some aspects related to modelling and conditioning factors. Tesis Doctoral, Agricultural University Wageningen, $160 \mathrm{p}$.

De Ploey, J. (1981): Crusting and time-dependent rainwash mechanims on loamy soil. En Morgan, R.P.C. (Ed.): Soil Conservation: Problems and Prospects, Wiley, 139-152.

De Ploey, J. (1983): Rainfall simulation, runoff and soil erosion. Catena Supplement. 4, $214 \mathrm{p}$.

DeBoer, D.W. (1991): Conservation Tillage on a Silt Loam Soil with reduced pressure sprinkler irrigation. Applied Engineeering in Agriculture, 7, 5, 557-562.

DiXon, R.M. y PETERSEn, A.E. (1968): A vacuum system for accumulation runoff from infiltrometers. Soil Sci. Soc. Amer. Proc., 32 (1) 123-125). 


\section{A. CERDÀ}

DORTIGNAC, E.J. (1951): Desing and operation of Rocky Mountain Infiltrometer. USDA Forest Ser. Rock Mountain Forest and Range.Exp. St. paper 5., Ft. Collins Colorado. 68 p..

Dunne, T., Dietrich, W.E. y Brunengo, M.J. (1980): Simple, portable equipment for erosion experiments under artificial rainfall. Journal Agric. Eng. Res., 25, 161168.

EDWARDS, L.M. y BuRNEY, J.R. (1992): Sedimet fractions in interrill runoff under various conditions of ground cover compaction and freeze/thaw using a rainfall simulator. Canadian Agricultural Engineering, 34 (1) 33-40.

EGASHIRA, K. y NAKAI, S. (1987): Size distribution and wet density of sediment eroded under simulated rainfall. Soil Sci. Plant Nutr., 33 (3) 347-354.

Euuson, W.D. (1947): Soil erosion studies. II. Soil detachment hazard by raindrop impact. Agricultural Engineering, 28, 197-201.

ElWell, H.A. y MAKWANYA, H. (1980): Design and Calibration of a Rainfall Simulator Nozzle Assembly for Laboratory and Field Studies. Dep. of Conservation and Extension, Rhodesia, Research Bulletin Number 25, 34 pp.

EpemA, G.F. y RiezeBos, H.Th. (1983): Fall velocity of waterdrops at different heights as a factor influencing erosivity of simulated rain. En J. De Ploey (Ed.): Rainfall Simulation, runoff and Soil Erosion, Catena supplement, 4, 1-17.

EPSTEIN, E., y GRANT, W.J. (1966): Desing construction and calibration of a laboratory rainfall simulator. Univ. of Maine Tech. Bull. $\mathrm{n}^{\Omega} 22$.

ERpul, G., Gabriels, D. y Janssens, D. (1998): Assessing the drop size distribution of simulated rainfall in a wind tunnel. Soil and Tillage Research, 45, 455-463.

Essery, C.I., WILCOCK, D.N. y MCCLEAN, W. (1987): A computer-based datalogging system used to investigate the infiltration process under natural rainfall conditions. Hydrological Processes, 1, 283-292.

Evans, R.G., SMITH, C.J., MrtCheld, P.D. y NewTon, P.J. (1990): Furrow infiltration on nontilles beds with cracking soils. Journal of Irrigation and Drainage Engineering, 116 (5) 714-33.

FARRES, P. (1978). The role of time and aggregate size in the crusting process. Earth Surface Processes, 3, 243-54.

FARRES, P. y CoUSEN, S. (1985): An improved method of aggregate stability measurement. Earth Surface Processes and Landforms, 10, 321-329

Farres, P.J. (1980): Some Observations on the Stability of Soil Aggregates to Raindrop Impact. Catena, 7, 223-231.

FARres, P.J. (1987): The dynamics of rainsplash erosion and the role of soil aggregate stability. Catena, 14, 119-30.

FLoYd, C.N. (1981): A Movile Rainfall Simulator for Small Plot Field Experiments. J. agric. Engng. Res., 26, 307-314. 
SIMULADORES DE LLUVIA Y SU APLICACIÓN A LA GEOMORFOLOGÍA. ESTADO DE LA CUESTIÓN

FOSTER, G.R., EPPERT, F.P. y MEYER, L.D. (1979): A programmable rainfall simulator for field plots. Proceedings of the rainfall simulator workshop, USDA-SEA , ARS-W-10/july 1979, 45-63.

Foster, G.R., Neibling, W.H. y NatTerman, R.A. (1982): A programmable rainfall simulator. ASAE paper $\mathrm{n}^{\mathrm{O}}$ 82-2570.

FrANCIS, C.F. (1990): Soil Erosion and Organic Matter Losses on Fallow Land: A Case Study from South-East Spain. En Boarman, J., Foster, I.D.L. y Dearing, J.A. (Eds.): Soil Erosion on Agricultural Land, 331-338.

Francis, C.F. y Thornes, J.B. (1990). Matorral: Erosion and Reclamation. En Albadalejo, J., Stocking, M.A. y Diaz, E. (Eds.): Degradacion y Regeneración del suelo en condiciones ambientales mediterráneas, 86-115.

Francis, C.F. y THORNEs, J.B. (1990). Runoff hydrografs from Three Mediterranean Vegetation Cover Types. En J.B Thornes (Ed.): Vegetation and Erosion, 365-384.

Gabriels, D. y DeBoodT, M. (1975): A rainfall simulator for erosion studies in the laboratory, Pedologie., 25, 80 86.

GEEDES, N. y DunkerLy, D. (1999): The influence of organic litter on the erosive effects of raindrops and of gravity drops released from desert shrubs. Catena, 36, 303-313.

GERITS, J.J.P. (1991): Pbysico-chemical tresholds for sediment detachment, transport and deposition. Tesis doctoral inedita. Universiteit van Amsterdam, 186 pp.

GHADRI, H. y PAYNE, D. (1977): Raindrop impact stress and the breakdown of soil crumbs. Journal of Soil Science, 28, 247-256.

GLANVILLE, S.F. y SMITH, G.D. (1988): Aggregate brekdown in clay soils under simulated rain and effects on Infiltration. Aust. J. Soil Res., 26, 111-120.

GREEN, D.M. (1998): Recreational impacts on erosion and runoff in a central Arizona riparian area. Journal of Soil and Water Conservation, 53, 38-42.

GREENE, R.B.S. and SAWTELL, G.R. (1992): A collection system for measuring runoff and soil erosion with a mobile rainfall simulator on sealed and stony red earth soils. Aust. J. Soil Res., 30, 457-463.

GRIERSON, I.T. y OADES, J.M. (1977): A rainfall simulator for field studies of runoff and soil erosion. J. Agr. Engr. Res., 22, 37-44.

GRIEvE, I.C. (1979): Soil aggregate stability tests for the geomorphologist. BGRG, Tecbnical Bulletin, 25.

GuNN, R. y KINZER, G.R. (1949): Terminal velocity of water droplets in stagnant air. Journal of metereology, 6, 243-248.

HALDER, J. (1989): Comparison between surface applied and incorporated wheat straw mulch for water erosion control. Msc thesis, Silsoe College, Silsoe, Inglaterra.

HAMON, W.R. (1979): Infiltrometer using simulated rainfall for infiltration research. Proceedings of the rainfall simulator workshop, USDA-SEA , ARS-W-10/july $1979,54-60$. 
HAssel, J. y Richter, G. (1988): Die Niederschlagsstruktur des Trierer Regensimulators. Mitteilgn. Dtsch. Bondenkundl. Gesellsch., 56, 93-96.

HERLILHY, T.E. (1990): Distribution patterns and rates for a fixed-nozzle, solen oidcontrolled variable-intensity, rainfall simulator. ASE paper 90-2512, 22 pp.

Hignett, C.T., Gusli, S., CASs, A. and Besz, W. (1995): An automated laboratory rainfall simulation system with controlled rainfall intensity, raindrop energy and soil drainage. Soil Technology, 8, 31-42.

HiLls, R.C. (1970): The determination of the infiltration capacity on field soils using the cylinder infiltrometer. B.G.R.G., Technical bull., 3, Goebooks Ltd., Norwich, 24 p.

Hinkle, S.E. (1990): The Akron USDA-Agricultural Research Sevice Portable BoomMounted Rainfall SImulator. TRANSACTIONS of the ASAE., 33 (3) 818-820.

Hino, M., FujtTA, K. y ShuTTo, H. (1987): A laboratory experiment on the role of grass for infiltration and runoff processes. Journal of Hydrology, 90, 303-325.

Hino, M., OdAKA, Y., NADAOKA, K. y SATO, A. (1988): Effect of initial soil moisture content on the vertical infiltration process-a guide to the problem of runoffratio and loss. Journal of Hydrology, 102, 267-284.

Hirschi, M.C., Barnhisel, R.I., Ruetten, M., Leopold, M. y Williams, R.G. (1981): Portable rainfall simulator for erosion studies. ASAE Paper-80-2058, 15 p.

Hirschi, M.C., Mitchell, J.K., Freezor, D.R. y Lesikar, B.J. (1990): MicrocomputerControlled Laboratory Rainfall Simulator. TRANSACTIONS of the ASAE, 33 (6) 1950-1953.

HODGES, W.K. y BRYAN, R.B. (1982): The influence of material behaviour on runoff initiation in the Dinosaur Badlands, Canada. En Bryan, R. y Yair, A. (1982): Badland Geomorpbology and Piping, 13-46. Geo-Books, Cambridge, University Press, 408 pp.

Hofmann, L. y RiEs, R.E. (1991): Relationship of soil and plant characteristics to erosion and runoff on pasture and range. Journal of soil and water conservation, 46 (2) 143-147.

Holland, M. E. (1969): Desing and testing of a rainfall system. Col. State Univ. Exp. Sta. CER 69-70 MEH 21 Ft. Collins,

HolTAN, H.N. (1961): A concept of infiltration estimates in watershed engineering. ARS-41-51, USDA Agricultural Research Service, 25 p.

Holtan, H.N. y KiRKPATRICK, M.H. (1950): Rainfall infiltration and hydraulics of flow in runoff computations. Trans. Amer. Geogh. Union, 31 (5) 771-779.

HORTON, R.E. 1939. Analysis of runoff-plat experiments with varying infiltration capacity. In Trans. Amer. Geoph. Union, 693-711.

Huang, Ch. y Bradford, J.M. (1993): Application of a Laser Scanner to Quantify Properties of Soil Crusts. Catena Suplement, 24, 129-139.

HubBaRD, R.K., Williams, R.G. y Erdman, M.D. (1989): Chemical Transport from Coastal Plain Soils Under Simulated Rainfall: I. Surface Runoff, Percolation, 
SIMULADORES DE LLUVIA Y SU APLICACIÓN A LA GEOMORFOLOGÝA. ESTADO DE LA CUESTIÓN

Nitrate, and Phospate Movement. TRANSACTIONS of the ASAE, 32 (4) 12501257.

Hubbard, R.K., Williams, R.G., Erdman, M.D. y Marti, L.R. (1989): Chemical Transport from Coastal Plain Soils Under Simulated Rainfall: II. Movement of Cyanozime, Sulfometuron-Methyl, and Bromide. Transactions of the ASAE., 32 (4) 1239-1249.

Hudson , N. (1964) Field Measurements of accelerated soil erosion in localized areas. Rhodesia Agricultural Journal, 31, 3, 46-48.

Hudson, N. (1963): Raindrop characteristics in south central United States. Rbodesian Jour. Agr. Res., 1, 6-11.

HuDson, N. (1964): A review of artificial rainfall simulators. Research Bull., 7, Department of Conservation and Extension Zimbabwe.

Hudson, N. (1965): The influence of rainfall on the mechanics of soil erosion. M. Sc. Thesis, University of Cap Town..

IMESON, A. C. (1977): A simple field-portable rainfall simulator for difficult terrain. Earth Surface Processes, 2, 431-36.

Imeson, A.C. Y Jungerius, P.D. (1976): Aggregate Stability and Colluviation in the Luxembourg Ardennes; An Experimental and Micromorphological Study. Earth Surface Processes, 1, 259-271.

IMESON, A.C., Kwaad, F.J.P.M. y Verstraten, J.M. (1982): The relationship of soil physical and chemical properties to the development of badlands in Morocco. En BRYAN, R.B. y YAIR, A. (Eds.): Badland Geomorphology and Piping, 47-70

Imeson, A.C., Verstraten, J.M., Van Mulligen, E.J. y Sevink, J. (1992): The Effect of Fire and Water Repellency on Infiltration and Runoff under Mediterranean Type Forest. Catena, 19, 345-361.

IzZARD, C.F. (1944): The surface profile of overland flow. Trans. Amer. Geogh. Union, Papers Hydrology, 693-711.

JOHNSON, C.W. y GORDON, N.D. (1988): Runoff and erosion from rainfall simulator plots on sagebrush rangeland. Transactions of the ASAE., 31 (2) 421-27.

JOHNSON, M.G. y BESCHTA, R.L. (1981): Seasonal Variation of Infiltration Capacities of Soils in Western Oregon. USDA Pacific Northwest Forest and Range Experimental Station 1363 Paper, 8 p.

Johnston, H.T., El-Sawr, E.M. y Cochrane, S.R. (1980): A study of the infiltration characteristics of undisturbed soil under simulated rainfall. Earth Surface Pocesses, 5, 159-174.

Jones, D.M.A. (1959): The shape of raindrops. Illinois State Water Survey, no. 77.

JorgEnSEN, D.W. y GARDNER, T.W. (1987): Infiltration Capacity of Disturbed Soils: Temporal Change and Lithologic Control. Water Resources Bulletin, 23, 6 , 1161-1172.

KAINZ, M. y RICHTER, G. (1991): Der Weihenstephaner Schwenkdüsenregner. Z. Pflanzenernähr. Bodenkd. 
Kamalu, C. (1994): The effects of slope length and inclination on the separate and combined actions of rainsplash and runoff. En Rickson, R.J. (Ed..): Conserving Soils Resources. European Perspectives, 143-149. CAB International. Cambridge

KAMPHORST, A. (1987): A small rainfall simulator for determination of soil erodibility. Netherlands Journal of Agricultural Soil Science, 35, 407-415.

KAPENZI, A. (1994): Effect of grass strip width on the control of soil erosion from steep slopes. Msc Thesis, Silsoe College, Silsoe, Inglaterra. (http://dove.geog.le.ac.uk/sgs4/www/PUBLIC/bgrg/records/recgs.htm).

KARL, J. and TOLDRIAN, H. (1973): Eine transportable Beregnungsanla.ge für die Mssung von OberflächenabfluB und Bodenabtrag. Wasser and Boden, 25, 6365.

KaY, M.E. y Loch, R.J. (1978): A modified Meyer rainfall simulator. Proc. Conf. on Agr. Engr. Toowomba, Australia., 78-81.

KAZó, B. (1966): A talajok vizgazdálkodási tulajdonságainak meghatórozása mesterséges esözteteö Készülékkel (The determination of the features of the watersupplies of soils by rainfall simulator). Agrokémia és talajtan, 15 (2) 239-252.

KEREN, R. (1989): Water-Drop Kinetic Energy Effect on Water Infiltraion in Calcium and Magnesium Soils. Soil Sci. Soc. Am.J., 53 (6) 1624-1628.

KERÉNYI, A. (1981): A study of the dynamics of drop erosion under laboratory conditions. En Erosion and Sediment Transport Measurement, IAHS Publication 133, 365-372.

KInELl, P.I.A. (1974): Splash erosion: some observations on the splash-cup technique. Soil Sci. Soc. Am. Proc., 38, 657-70.

KInELL, P.I.A. (1990): The mechanims of raindrop induced flow transport. Australian J. Soil Res., 34, 715-720.

KLEIJN, W.B., OsTER, J.D. y CoOK, N. (1979): A rainfall simulator with nonrepetitious movement of drop outlets. Soil Sci. Soc. Am. J., 46., 93-99.

Kranz, W.L., Kanwar, R.S. y PEDERSON, C.E. (1998): Collection and monitoring of one-meter cubic soil monoliths for leaching studies. Transactions of the ASAE, 41, 333-344.

LAflen, J.M., Baker, J.L., Hartwig, R.O., Buchele, W.F. Y Johnson, H.P. (1978): Soil and Water Loss from Conservation Tillage Systems. Transactions of the ASAE, 21 (5) 881-885.

LANE, L.J. (1986): Erosion on rangelands: emerging technology and data base. Proceedings of the rainfall simulator workshop. Sociaty for Range Management, Tucson, Arizona, 68 pp.

LatTanzi, A.R., Meyer, L.D. y Baumgardner, M.F. (1974): Influences of mulch rate and slope steepness on interril erosion. Soil Sci. Soc. Am. Proc., 38, 946-50.

LAWS J.O. y PARSONS, D.A. (1943): The relationship of raindrop size to intensity. Trans. Am. Geophys. Union, 24, 452-460. 
SIMULADORES DE LLUVIA Y SU APLICACIÓN A LA GEOMORFOLOGÍA. ESTADO DE LA CUESTTÓN

LAws, J.O. (1941): Measurement of the fall-velocity of water drops and rain drops. Trans. Am. Geophys. Union, 22, 709-721.

LE BissonaIs, Y. (1989): Analyse des processus de microfissuration des agrégats à l'humectation. Science du Sol, 27, 187-199.

LEATHAN, P. y RIESBOL, H.S. (1950): Infiltration and retention test as related to spillway design floods. Trans. Amer. Geogh Union, 31 (2) 234-242.

Levy, G.J., Levin, J. y Shainberg, I. (1997): Prewetting rate and aging effects on seal formation and interrill soil erosion. Soil Science, 162, 131-139.

LOCH, R. y DONOLLAN, T.E. (1983): Field rainfall simulator studies on two clays of the Darling Downs, Queensland. I. The effect of plot size and tillage orientation on erosion processes and runoff and erosion rates. Australian Journal of Soil Research, 21, 47-58.

Low, A.J. (1954): The study of soil structure in the field and the laboratory. Journal of Soils Science, 5, 1-57.

LuK, S.-H. (1985): Effect of Antecedent Soil Moisture Content on Rainwash Erosion. Catena, 12, 129-139.

Luk, S.H., ABrahams, A.D. y Parsons, A.J. (1986): A simple rainfall simulator and trickle system for hydro-geomorphological experiments. Physical Geography, 7, 344-356.

LUSBY, G.C. (1977): Determination of runoff and sediment by rainfall simulation. En Toy, T.J. (Ed.): Erosion: research techniques, erodibility and, sediment yield. Geobooks Ltd. Norwich, England, 19-39.

Lyles L., DisRub, L.A. y WoOdruff, N.P. (1969): Effects of soil physical properties, rainfall characteristics, and wind velocity on clod disintegration by simulated rainfall. Soil Sci. Soc. Am. Proc., 33 (2) 302-306.

MaleKuTI, A. y GIFFORD, G.F. (1978): Natural vegetation as a source of diffuse salt within the Colorado River Basin. Water Res. Bulletin, 14, 195-205.

MARIEN, J.L. y Vandewiele, G.L. (1986): A point rainfall generator with internal storm structure. Water Resources Research, 22 (4) 475-482.

Marsh, B. A' B. (1974): Overcoming run-off plot isolation. Agric. Eng, Con. Proc. Sydney, Vol. 1. 104-114.

MARSTON, D. ( 1978): The use of simulated rainfall in assessing the erodibility of various stubble management practices. Proc. Conf. on Agr. Enger., Toowomba, Australia.

MARSTON, D. (1982): A rainfall simulator for field determination of relative erosion potentials. Journal of the Soil Conservation Service of N.S.W., 38, 31-39.

MARTínez, M., LANE, L.J. y FogEL, M.M. (1979): Experimental investigation of soil detachment by raindrop impact. En Proceedings of the rainfall simulator workshop. USDA-SEA, ARS-W-10/july 1979, 153-155.

Mazurak, A.O. y Mosher, P.N. (1968): Detachment of soil particles in simulated rainfall. Soil Sci. Soc. Am. Proc., 32, 716-719. 
McCalla, T.M. (1944): Water drop method of determining stability of soil structure. Soil Science, 58, 117-121

McGhie, D.A. y Posner, A.M. (1981): The effect of plant top material on the water repellence of fires sands and water repellent soils. Aust.J. Agrec. Res., 32, 609620.

MCKERSIE, W.M. (1985): The design of a rotating nozzle rainfall simulator and the testing with selected nozzles. Bsc thesis, Silsoe College, CIT.

MCKERSIE, W.M. (1995): The design of a rotating nozzle rainfall simulator, and the testing with selected nozzles. Bsc Thesis, Silsoe College, Silsoe, Inglaterra.

MCQUEEN, I.S. (1963): Development of a hand portable rainfall-simulator infiltrometer. U.S. Geol. Surv. Circ. 482, 16 p.

Medalus (1993): Medalus II report. Silsoe College, Silsoe, Inglaterra.

MEEWING, R.O. (1969): Infiltration and soil erosion as influences by vegetation and soil in northern Utah. Journal of Range Management, 23 (3) 185-188.

MELVILLE, N. (1992): The influence of grass density on the effectiveness of contour grass strips. Msc Thesis, Silsoe College, Silsoe, Inglaterra.

MeYer, L.D. (1960): Use of the rainulator for runoff plot research. Soil Sci. Soc. Am. Proc., 24, 4, 319-22.

MEYER, L.D. (1979): Current rainfall simulators and research activities. Proceedings of Rainfall Simulator Workshop, Tucson, Ariz, USDA-SEA, Agric. Reviews and Manuals ARM-W-10, 156-157.

MEYER, L.D. (1979b): Current rainfall simulators and research activities. Proceedings of Rainfall Simulator Workshop, Tucson, Ariz, USDA-SEA, Agric. Reviews and Manuals ARM-W-10, 156-157.

MEYER, L.D. y HARMON, W.C. (1979): Rainfall simulator for evaluating erosion rates and sediment sizes for row sideslopes. Transactions of the ASAE, 22 (1) 100-103.

MeYer, L.D. y MCCune, D.L. (1958): Rainfall simulator for runoff plots. Agricultural Engineering, 39 (10) 644-648.

MiLLER, W.P. (1987): A Solenoid-operated variable intensity rainfall simulator. Soil Sci. Soc. Am. J., 51, 832-834.

Mokyersons, J. (1983): Measurement of Splash-Saltation fluxes under obleque rain. Catena Supplement, 4, 19-31.

Moore, I.D., Hirschi, M.C. y BArfield, B.J. (1983): Kentucky Rainfall Simulator. TRANSACTIONS of the ASAE, 26 (4) 1085-1089.

Morin, J., Goldberg, D. y Seginer I. (1967): A rainfall simulator with rotating disk. TRANSACTIONS of the ASAE, 10, 74-79 p.

MulLINGEN, E. J. VAN., (1990): Erosieonderzoek voor mais-, suikervbieten- end aardappelteelt. Rapport ROC-UvA, 17-25

Munn, D.A., Mclean, E.O., Ramirez, A. y Logan, T.J. (1973): Effect of soil, cover, slope, and rainfall factors on soil and phosphorus movement under simulated rainfall conditions. Soil Sci. Soc. Am. Proc., 37, 428-31. 
SIMULADORES DE LLUUIA Y SU APLICACIÓN A LA GEOMORFOLOGÍA. ESTADO DE LA CUESTIÓN

Munn, J.R. y Huntington, G.L. (1967a): A portable simulator for erodibility and infiltration measurements on ruggedze characteristics of sediment from agricultural soils. Journal of Soil and Water Conservation, 47, 107-111.

MunN, J.R. y Huntington, G.L. (1967b): A portable simulator for erodibility and infiltration measurements on ruggedze characteristics of sediment from agricultural soils. Journal of Soil and Water Conservation, 47 (1) 107-111.

Mutchler, C.H. y MoldenHAueR, W.C. (1963): Applicator for laboratory rainfall simulator. TRANSACTIONS of the ASAE, 6 (3) 220-222.

MuTCHLER, C.K. y HersmeIER, L.F. (1965): A review of rainfall simulators. Trans. Am. Soc. Agr. Engrs., 6, 220-222.

Nammah, H., Larsen, F.E., Mccool, D.K., Fritts, R. y Molnau, M. (1986): Mt. St. helens Volcanic ash: effect of incorparated and unincorporated ash of two particle sizes on runoff and erosion. Agriculture, ecosystems and environment, 15 , 63-72.

NASSIF, S.H. y WiLSON, E.M. (1975): The influence of slope and rain intensity on runoff and infiltration. Hydrol. sci. Bull., 20, 539-553.

Navas, A., Alberto, F., Machin, J. y Galan, J. (1990): Design and operation of a rainfall simulator for field studies of runoff and soil erosion. Soil Technology, 3, 385-397.

NEAL, J.H. (1938): The effect of the degree of slope and rainfall characteristics on runoff and soil erosion. Univ. of Missouri Agricultural Experiments Station Research Bulletin 280.

Neff, E.L. (1979): Simulator activities in Sidney, Montana. En Proceedings of the rainfall simulator workshop, USDA-SEA, ARS-W-10/july 1979, 160-161.

NEFF, E.L. (1982): Performance characteristics and field operation of two rainfall simulators. BLM-ARS Interagency Agreement YA-515-IA6-3 Report, USDA-ARS, $26 \mathrm{pp}$.

Neibling, W.H., Foster, G.R., Natterman, R.A., Nowlin, J.D. y Holbert, P.V. (1981): Laboratory and field testing of a programmable plot-sized rainfall simulator. Erosion and sediment transport measurement. IAHS Publication 133, 405-414.

Nichols, M.L. y SEXTON, H.D. (1932): A method of studing soil erosion. Agricultural Engineering, 13, 101-103.

Nishimura, T., NAKANo, M. y MryazaKi, T. (1993): properties of surface crusts of an Andisol and their effects on soil-hydrological processes. Catena Suplement, 24, 17-28.

NorTon, L.D. (1987): Micromorphological Study of Surface Seals Developed under Simulated Rainfall. Geoderma, 40, 127-140.

OGDEN-CB; van Es H.M. y Schindelbeck, R.R. (1997). Miniature rain simulator for field measurement of soil infiltration. Soil Sci. Soc. Am.J. 61 (4) : 1041-10 
A. CERDȦ

ONSTAD, C.A., RADKE, J.K. y YOUNG, R.A. (1981): An autdoor portable rainfall erosion laboratory Erosion and sediment transport measurement. IAHS Publication 133, 415-422.

OOsTwoud-Wijdenes, D.J. y ERGENZINGER, P. (1998): Erosion and sediment transport on steep marly hillslopes, Draix, Haute- Provence, Frnace; an experimental field study. Catena, 33, 179-200.

Ortiz de Zarate, A.R., Faci, J. y Aragües, R. (1991): Efecto de la pluviometría del riego por aspersión y de la coberturas del suelo sobre la infiltración del agua en el suelo de Monegros II. En Jornadas sobre Riegos y drenajes, Granada.

PARCKER, P.E. (1957): Intermountain infiltrometer. USDA Forest Service, Ogden, Utah., Intermont. Forest and Range Expt. Station, Misc. Publ., 14 p.

PARKER, R.S. y SCHUMM, S.A. (1982): Experimental studiy of drainge networks. En Bryan, R.S. y Yair, A. (De.): Badland Geomorpbology and Piping, 153-168.

Parsons, A.J., Abrahams, A.D. y Luk, S.G.(1990): Hydraulics of interrill overland flow on a semi-arid hillslope, Southern Arizona. Journal of Hydrology, 117, 255-273.

PARSONS, T. Y GREENE, M. (1994): Lechler axial con jet simulator. BGRG Rainfall simulator data base. (via internet:

http://dove.geog.le.ac.uk/sgs4/www/PUBLIC/bgrg/records/recgs.htm).

PEDERSON, R.N. y Du BoIs, B. (1986): Microprocessor Controlled, Variable Intensity, Variable Timing, Rainfall Simulator. Conference on Agricultural engineering (Adelaide), 6 pp.

Pelegrin, F., Camps, M., Moreno, F. y López, J. (1989): Estudio crítico del control de la intensidad de llluvia en un simulador para el cálculo de la infiltración de agua en el suelo. Asociación Nacional de Ingenieros Agrónomos, 24 Feria Técnica Internacional de la Maquinaria Agricola, Zaragoza, 135-141.

PEREIRA, H.C. (1956): Structure in tropical soils. Journal of Soil Science, 7 (1) 68-74.

PEREz, F.L. (1998): Conservation of soil moisture by different stone covers on alpine talus slopes (Lassen, California). Catena, 33, 155-177.

Peterson , A.E. y BuBEnzer, G.D. (1984): Intake rate - sprinkler infiltrometer. En Methods of Soil Analysis. Amer. Soc. Agron. Monograph.

Peterson, R.J. (1977): Laboratory simulation of soil erosion. Tesis Doctoral no publicada, Colorado State Univ. 70 p.

PoEsEn, J. (1984): The influence of slope angle on infiltration rate and hortonian overland flow volume. Z. Geomorph. N.F. Suppl.-Bd., 49, 117-131.

Poesen, J., Ingelmo-SÁnchez, F. y Mucher, H. (1990): The hydrological response of soil surfaces to rainfall as affectes by cover and position of rock fragments in the top layer. Earth Surface Processes and Landforms, 15, 653-671.

POESEN,J.W.A. y VAN, WeSEMAEL, B. (1993): Effects of rock fragments on the structural collapse of tilled topsoils during rain. In E. Derbyshire, Dijkstra, T. y 
SIMULADORES DE LLUVIA Y SU APLICACIÓN A LA GEOMORFOLOGÍA. ESTADO DE LA CUESTIÓN

Smalley, I. J. (Eds.): Genesis and properties of collapsible soils. Kluwer Academic publishers, Dordrecht, Nato Advanced Science Institute Series, C, Mathematical and Physical Sciences, Vol. 468: 333-343.

PONCE, S.L. (1975): Examination of a nonpoint source loading function for the Mancos Shale wildlands of the Price River Basin, Utab. Tesis Doctoral, Dep. of Civil and Environmental Engineering, Utah State University.

Ponce, S.L. y Hawkins, R.H. (1978): Salt pickup by overland flow in the Price River Basin, Utah. Water Resources Bulletin, 14 (5) 1187-1200.

Rai, K.D., RANey, W. A. y VANDERFord, H. B. (1954): Some physical factors that influence soil erosion and the influence of aggregate size and stability on growth of tomatoes. Proc. Soil Sci. Soc. Amer.J. 19 (2) 221-232.

Rawits, E., Margolin, M. y Hillel, D.I. (1972): Un improved variable-intensity sprinkling infiltrometer. Soil Sci. Soc. Amer. Proc., 36 (3) 533-535.

Rhoton, F.E., LindBo, D.L. y Römkens, M.J.M. (1998): Iron oxides erodibility interactions for soils of the Memphis catena. Soil Sci. Soc. Am.J., 62, 1693-1703.

RiEzEBOS, H.T. Y SEYHAN, E. (1977): Essential conditions of rainfall simulation for laboratory water erosion experiments. Earth surface processes, 2, 185-190.

RodríguEZ, O.S. (1997): Hedgerows and mulch as soil conservation measures evaluated under field simulated rainfall. Soil Technology, 11, 79-93.

RÖMKENS, M.J.M. (1979): A rainfall simulator for small laboratory samples. Proceedings of the rainfall simulator workshop, Tucson, AZ. USDA-SEA ARMW-10, 162.

Römkens, M.J.M., Glenn, L.F. y Nelson. D.W. (1975): A laboratory rainfall simulator for infiltration and soil detachment studies. Soil Sci. Soc. Amer. Proc., 39 (1) 158-160.

Rose, G.W. (1960): Soil detachment caused by rainfall. Soil Science, 89, 28-35.

Roth,C.H., MEYer, B. y Frede, H.-G. (1985): A portable rainfall simulator to study factors affecting runof, infiltration and soil loss. Catena, 12, 79-85

Rowe, P.B. (1940): The construccion, operation and use of the North Fork Infiltrometer. U.S. Flood Control Coord. Comm., Misc. Publ., 1, Caif. Forest and Ra. Expt. Station, Misc. Publ., 1.

Ruiz Flaño, P. (1993): Procesos de erosión en campos abandonados del Pirineo. Geoforma Ediciones, Logroño, 191 pp.

SÁNCHEZ, C.E. y WOOD, M.K. (1987): The relationship of soil surface roughness with hydrologic variables on natural and reclaimend range land in New Mexico. Journal of Hydrology, 94, 345-54.

SANRoque, P., Rubio, J.L. y SÁnCHEZ, J. (1984): Simulador de lluvia para el estudio de la erosionabilidad del suelo en laboratorio. I Congreso Español de Geologia, I, 783-794. 


\section{A. CERDÀ}

SARdo, V., Vella, P. y Zimbone, S.M. (1994): Preliminary investigation of runoff using a rainfall simulator. En Rickson, R.J. (De.): Conserving Soils Resources. European Perspectives, 136-142. CAB International. Cambridge.

SAVAT, J. (1977): The hydraulics of sheet flow on a smooth surface and the effect of simulated rainfall. Earth Surface Processes, 2, 125-40.

ScHMIDT, R.-G. (1983): Ein Regensimulator für Feldversuche. Wasser und boden, 4 , 179-182.

Schultz, J.P., Jarret, A.R. y Hoover, J.R. (1985): Detachment and splash of cohesive soil by rainfall. Transactions of the ASAE, 28 (6) 1878-1884.

SCHulz, E.F. y Yevjevich, V. (1970): Experimental investigation of small watershed floods. Colorado State University, Department of Civil Engineering, Report No. CER 69.70. ERS-VY38.

SCoging, H. (1982): Spatial variations in infiltration runoff and erosion on hillslopes in semi-arid spain. In Bryan, R. y Yair, A. (Eds.): Badland geomorpbology and piping, 89-112.

Scoging, H.M. y Thornes, J.B. (1980): Infiltration characteristics in a semiarid environment. IAHS Publication, 128, 159-168.

SELBY, M.J. (1970): Design of a hand-portable rainfall simulating infiltrometer, with trial results from the Oturira catchment. Journal of bydrology, 9 (2) 117-132.

SHEDDEN, J. 1980. Cover in soil erosion process. Msc Thesis, Silsoe College, Silsoe, Inglaterra.

SHIRner, D.S., ABNER, C.H. y MANN, L.K. (1977): Rainfall simulation for enviromental application. Oak Ridge National Lab. Envi. Sci. Div. Publ. No. 1067.

SiEmENS, J.C. y OSCHWALD, W.R. (1978): Corn-soybeans tillage systems: erosion control, effects on crop production, costs. Transactions of the ASAE., 21, 293-302.

Simaton, J.R., Johnson, C.W., NyHAN, J.W. y Romney, E.M. (1986): Rainfall Simulation on Rangeland Erosion Plots. En Lane, L.J. (Ed.): Erosion on rangelands: emerging technology and data base. Proceedings of the rainfall simulator workshop. Sociaty for Range Management, Tucson, Arizona, 11-17.

Simón, M., Iriarte, A., Guardiola, J.L., Barahona, E. y García, I. (1994): SoilInfiltration rate under different vegetation cover using a rain simulator of variable intensity. In Rodríguez, A., Jiménez, C. y Tejedor, M.L. (Eds.): The soil as a strategic resource: degradation processes and conservation measures, 177-188, Geoforma Ediciones, Logroño.

SlatTERY, M.C. y BRYAN, R.B. (1994): Surface seal development under simulated rainfall on an actively eroding surface. Catena, 22, 17-34.

SMITH, D.D. y WischmeIER, W.H. (1962): Rainfall erosion. Advances in agronomy, 14, 109-148.

Smith, H.J.C., Levy, G.J. y ShainBerg, I. (1990): Water-Droplet Energy and Soil Amendments: Effect on Infiltration and Erosion. Soil Sci. Soc. Am. J., 54 (4) 1084-1087. 
SIMULADORES DE LLUVIA Y SU APLICACIÓN A LA GEOMORFOLOGÍA. ESTADO DE LA CUESTIÓN

SpILHAUs, A. F. (1948): Drop size, intensity, and radar echo of rain. Journal of metereology, 5, 161-164.

STEINHARDT, R. y HiLLEL, D.I. (1966): A portable low-intensity rain simulator for field and laboratory use. Soil Sci. Soc. Amer. Proc., 30, 661-662.

Sutherland, R.A. y Bryan, R.B. (1988): Solute sources and transport in an ephemeral badland catchment, southeastern Alberta, Canada. Can. J. Earth Sci., 167-181 (1988).

SWANSON, N.P. (1965): Rotanting boom rainfall simulator. Transactions of the ASAE, 8 (1) $71-72$.

TECK, F.H. (1993): A preliminary validation and aplication of erodibility indices for soils in Sarawak, Malaysia. Msc. Silsoe College, Silsoe, Inglaterra.

Ternan, J.L., Williams, A.G. y GonzÁlez, M. (1994): Soil properties and gully erosion in the Guadalajara province, Central Spain. En Rickson, R.J. (Ed.): Conserving Soils Resources. European Perspectives, 56-69. CAB International. Cambridge.

Tossell, R.W., Dickinson, W.T., Rudra, R.P. y WallL, G.J. (1987): A Portable Rainfall Simulator. Canadian Agricultural engineering, 29 (2) 155-162.

TURNER, A. K. (1965): The simulation of rainfalls for studies in overland flow. Journal of the Institution of Engineers, 9-15.

Turner, A.K. y LANGLORD, K.A. (1969): A rainfall simulator and associated facilities for hydrologic studies. J. Aust. Inst. Agr. Sciences, 35 (2) 115-199.

URESTI GIL, J. (1990): Testing and calibration of a rotatin nozzle rainfall simulator. Msc thesis, Silsoe College, Silsoe, Inglaterra.

VILENSkil, (1953): Citado en Vershimin, P.V. (1971): The background o soil structure. Editado por S. Dolgov, Jerusalem (1971).

Walker, P.H., Hutka, J., Moss, A.J. y Kinel, P.I.A. (1977): Use of a versatile system for soil erosion studied. Soil Sci. Soc. Am..J., 41, 609-612.

WAN, Y., EL-Swaify, S.A. y SuTHERLAND, R.A. (1996): Partitioning interrill splash and wash dynamics: a novel laboratory approach. Soil Technology, 9, 55-69.

Wierda, A., VeEn, A.W.L. y HuTJes (1989): Infiltration at the Tai rain forest (Ivory Coast): Measurement and modelling. Hydrological Processes, 3, 371-382.

Wilcox, B.P., WoOd, M.K., Tromble, J.T. y Ward, T.J. (1986): A hand-portable single nozzle rainfall simulator designed for use on steep slopes. Journal of Range Management, 39 (4), 331-5.

Williams, A., Ternan, J.L., Elmes, A., del Tanago, G. y Blanco, R. (1995): A field study of the influence of land management and soil properties on runoff and soil loss in central Spain. Environmental Monitoring and Assessment, 37, 1528.

Wilm, H.G., (1943): The application and measurement of artificial rainfall on types FA and F infiltrometers. Trans. Amer. Geopbys. Union., 3, 480-484. 
Wilson, B.N. y Rice, C.T. (1987): Large-Scale Laboratory Apparatus for Erosion Studies. ASAE Paper, 19 p.

Wilson, B.N. y Rice, C.T. (1990): An indoor soil erosion research facility. Journal of Soil and Water Conservation, 45 (6) 645-648.

WischmeIER, W. H. (1959): A rainfall erosion index for a universal loss equation. Proc. Soil Sci. Soc. Amer., 23, 246-249.

WIschmeIER, W.H. y SMITH, D.D. (1978): Predicting Rainfall Erosion losses. USDA Agricultural Handbook, 537 p.

WOLFGARTEN, H.-J. and FrANKEN, H. (1988): Bestimmung der Erosionsgefährdung verschiedener Anbauverfahren (z.B. bei Zuckerrüben) mit Regensimulation Bonner Regensimulator. Mitt. Dtsch. Bodenkdl. Ges., 56, 43-46.

WRIGHT, J.S. (1991): An assessment of the potential of vertical multching for soil erosion control. Msc thesis. Silsoe College. Inglaterra.

YAIR, A. y LAVEE, H. (1976): Runoff generative process and runoff yield from arid talus mantled slopes. Earth Surface Processes and Landforms, 1, 235-247.

YounG, R.A. (1979): Rainfall simulator activities at the North Central soil conservation research laboratory, Morris, Minessota. En Proceedings of the rainfall simulator workshop, USDA-SEA, ARS-W-10/july 1979, 171-172- 172.

ZEGELIN, S.J. y WHITE, I. (1982): Design for a fields sprinkler infitrometer. Soil Sci. Soc. Am.J., 46, 1129-1133. 